\title{
Generalized prolate spheroidal wave functions for optical finite fractional Fourier and linear canonical transforms
}

\author{
Soo-Chang Pei and Jian-Jiun Ding \\ Department of Electrical Engineering, National Taiwan University, Taipei, Taiwan 10617
}

Received May 13, 2004; revised manuscript received September 14, 2004; accepted September 16, 2004

Prolate spheroidal wave functions (PSWFs) are known to be useful for analyzing the properties of the finiteextension Fourier transform (fi-FT). We extend the theory of PSWFs for the finite-extension fractional Fourier transform, the finite-extension linear canonical transform, and the finite-extension offset linear canonical transform. These finite transforms are more flexible than the fi-FT and can model much more generalized optical systems. We also illustrate how to use the generalized prolate spheroidal functions we derive to analyze the energy-preservation ratio, the self-imaging phenomenon, and the resonance phenomenon of the finitesized one-stage or multiple-stage optical systems. (†) 2005 Optical Society of America

OCIS codes: $070.2580,070.2590,350.6980$.

\section{INTRODUCTION}

The Fourier transform (FT) is defined as

$$
\begin{aligned}
\text { FT: } \quad F(\omega) & =(2 \pi)^{-1 / 2} \int_{-\infty}^{\infty} \exp (-j \omega x) f(x) \mathrm{d} x, \\
x, \omega & \in(-\infty, \infty) .
\end{aligned}
$$

Notice that, in both the space and the frequency domains, the intervals are $(-\infty, \infty)$. However, owing to the finite size of the implementing components, the infinite interval is always impossible. In practice, we perform the finite Fourier transform (fi-FT) instead of the FT:

$$
\text { fi-FT: } \quad \tilde{F}(\omega)=(2 \pi)^{-1 / 2} \int_{-T}^{T} \exp (-j \omega x) f(x) \mathrm{d} x .
$$

The space interval is $x \in[-T, T]$, and the frequency interval is $\omega \in[-\Omega, \Omega]$. Since the intervals are finite, after the fi-FT is done, some energy is lost. For the original FT, the energy-preservation property is satisfied:

$$
\int_{-\infty}^{\infty}|F(\omega)|^{2} \mathrm{~d} \omega=\int_{-\infty}^{\infty}|f(x)|^{2} \mathrm{~d} x .
$$

However, for the fi-FT, the energy ratio of $\widetilde{F}(\omega)$ to $f(x)$ is smaller than 1:

$$
0<\text { energy preservation ratio }=\frac{\int_{-\Omega}^{\Omega}|\tilde{F}(\omega)|^{2} \mathrm{~d} \omega}{\int_{-T}^{T}|f(x)|^{2} \mathrm{~d} x}<1 .
$$

Then one may ask under what condition the energy ratio is maximal. In 1961 Slepian and Pollak ${ }^{1}$ and Landau and Pollack ${ }^{2,3}$ found that these problems could be analyzed by prolate spheroidal wave functions (PSWFs).

PSWFs are the continuous functions that satisfy

$$
\begin{aligned}
\int_{-T}^{T} K_{F, \Omega}(\tilde{x}, x) \psi_{n, T, \Omega}(x) \mathrm{d} x & =\lambda_{n, T, \Omega} \psi_{n, T, \Omega}(\tilde{x}), \\
K_{F, \Omega}(\tilde{x}, x) & =\frac{\sin [\Omega(\tilde{x}-x)]}{\pi(\tilde{x}-x)} .
\end{aligned}
$$

Infinite functions can satisfy Eq. (5). We can sort them according to the values of $\lambda_{n, T, \Omega}$ 's:

$$
\begin{aligned}
1> & \lambda_{0, T, \Omega}>\lambda_{1, T, \Omega}>\lambda_{2, T, \Omega}>\cdots>0 \\
& \left(\text { all of } \lambda_{n, T, \Omega} \text { real) } .\right.
\end{aligned}
$$

We write $T$ and $\Omega$ in the subscripts because PSWFs and their eigenvalues are dependent on $T$ and $\Omega$. In the case in which the roles of $T$ and $\Omega$ are not emphasized, we will simplify the notations as $\psi_{n}(x)$ and $\lambda_{n}$.

PSWFs are orthogonal in the intervals of both $x$ $\in[-T, T]$ and $x \in(-\infty, \infty)$ :

$$
\begin{aligned}
& \int_{-T}^{T} \psi_{m}(x) \psi_{n}(x) \mathrm{d} x=\lambda_{n} \delta_{m, n}, \\
& \int_{-\infty}^{\infty} \psi_{m}(x) \psi_{n}(x) \mathrm{d} x=\delta_{m, n} .
\end{aligned}
$$

When $T \times \Omega \rightarrow \infty$, they are similar to HermiteGaussian functions:

$$
\begin{gathered}
\psi_{n}(x) \approx C_{n} \exp \left(-x^{2} / 2\right) H_{n}(x), \quad T \times \Omega \rightarrow \infty, \\
H_{n}(x): \text { Hermite polynomials. }
\end{gathered}
$$

The asymptotic approximation of PSWFs was discussed in Ref. 4.

To compute PSWFs numerically, we can set $x=k \Delta$ and $\tilde{x}=h \Delta$. Then Eq. (5) becomes 


$$
\begin{aligned}
R_{F, \Omega}[k, h]= & K_{F, \Omega}(k \Delta, h \Delta) \times \Delta \\
= & \frac{\sin [\Omega \Delta(k-h)]}{\pi(k-h)} \\
& \quad\left(m, n=-N_{0},-N_{0}+1, \ldots, N_{0}\right),
\end{aligned}
$$

where $\Delta=2 T N^{-1}$ and $N=2 N_{0}+1$. Then we define the tridiagonal matrix $\mathbf{T}$ as $^{5}$

$$
\begin{aligned}
& T[p, p-1]=T[p-1, p]=\beta_{p}, \quad T[p, p]=\alpha_{p} \\
& T[p, q]=0 \text { otherwise } \\
& \beta_{p}=2(p-1)(N-p+1) \\
& \alpha_{p}=[N+1-2 p]^{2} \cos (\Omega \Delta) \\
& \qquad p, q=1,2,3, \ldots, N
\end{aligned}
$$

Since $\mathbf{R}_{\mathbf{F}, \boldsymbol{\Omega}}$ commutes with $\mathbf{T}$ (i.e., $\mathbf{R}_{\mathbf{F}, \mathbf{\Omega}} \mathbf{T}=\mathbf{T R}_{\mathbf{F}, \boldsymbol{\Omega}}$ ) and $\mathbf{T}$ has no repeated eigenvalues, the eigenvectors of $\mathbf{T}$ are also the eigenvectors of $\mathbf{R}_{\mathbf{F}, \boldsymbol{\Omega}}$. It is known that the eigenvectors of a tridiagonal matrix are easier to solve. ${ }^{6}$ Thus we can use the tridiagonal matrix $\mathbf{T}$ to solve the eigenvector of $\mathbf{R}_{\mathbf{F}, \boldsymbol{\Omega}}$ and hence the spherical prolate function efficiently. We describe the numerical computation process for PSWFs as follows.

1. First, we calculate the determinants of the leading principal submatrices of $\mathbf{T}-\mu \mathbf{I}$ (denoted by $p_{r}(\mu)$, $r=1,2, \ldots, N)$ (from p. 300 of Ref. 6 ):

$$
\begin{aligned}
& p_{r}(\mu)=\left(\alpha_{r}-\mu\right) p_{r-1}(\mu)-\beta_{r}^{2} p_{r-2}(\mu) \\
& \quad\left[p_{1}(\mu)=\alpha_{1}-\mu \text { and defining } p_{0}(\mu)=1\right] .
\end{aligned}
$$

2. Then we use the bisection method together with the Sturm sequence property (see pp. 300-306 of Ref. 6) to solve the eigenvalues $\mu_{0}, \mu_{1}, \mu_{2}, \ldots, \mu_{N-1}$ of $\mathbf{T}$ from the equality $p_{N}\left(\mu_{n}\right)=0$. Notice that the eigenvalues of $\mathbf{T}$ are not the solutions of $\lambda_{n}$ 's in Eq. (5).

3. Then the eigenvectors of $\mathbf{T}$ can be solved from (see p. 316 of Ref. 6 ):

$$
v_{n}[1]=1, \quad v_{n}[r]=(-1)^{r-1} p_{r-1}\left(\mu_{n}\right)\left(\beta_{2} \beta_{3} \ldots \beta_{r}\right)^{-1} .
$$

Since $\mathbf{R}$ and $\mathbf{D}$ have the same eigenvectors, $\mathbf{v}_{\mathbf{n}}$ 's are also the eigenvectors of $\mathbf{R}$ in Eq. (9).

4. The eigenvalues $\lambda_{n}$ 's of the integral equation in Eq. (5) are close to the eigenvalues of $\mathbf{R}$ :

$$
\lambda_{n} \approx \tau_{n},
$$

where $\mathbf{R} \mathbf{v}_{\mathbf{n}}=\tau_{n} \mathbf{v}_{\mathbf{n}}, \mathbf{R}$ is defined in Eq. (9), and $\mathbf{v}_{\mathbf{n}}$ is derived in Eqs. (13).

5. Since $\mathbf{R}$ is obtained from sampling the integral equation in Eq. (5), its eigenvectors $\mathbf{v}_{\mathbf{n}}$ 's approximate the solutions of $\psi_{n}(x)$ (i.e., the PSWF) in Eq. (5):

$$
\begin{gathered}
\psi_{n}\left\{\left[k-2^{-1}(N+1)\right] \Delta\right\} \approx A_{n} v_{n}[k], \\
A_{n}=\lambda_{n}^{1 / 2}\left\{\sum_{n} v_{n}^{2}[n] \Delta\right\}^{-1 / 2} .
\end{gathered}
$$

We choose the normalized constant $A_{n}$ as above to satisfy Eqs. (7).
PSWFs are helpful for calculating the power-preservation ratio [see expression (4)] of the fi-FT. Since $\left\{\psi_{n}(x) \mid n \in Z^{+}\right\}$forms a complete eigenfunction set, any function $f(x)$ can be expanded as a linear combination of $\psi_{0}(x), \psi_{1}(x), \psi_{2}(x), \psi_{3}(x), \ldots$ :

$$
f(x)=\sum_{n=0}^{\infty} a_{n} \psi_{n}(x), \quad x \in[-T, T] .
$$

Then in expression (4),

$$
\begin{aligned}
& \int_{-\Omega}^{\Omega}|\widetilde{F}(\omega)|^{2} \mathrm{~d} \omega=\int_{-\Omega}^{\Omega} \tilde{F}(\omega) \tilde{F}^{*}(\omega) \mathrm{d} \omega \\
& =\frac{1}{2 \pi} \int_{-\Omega}^{\Omega} \int_{-T}^{T} \exp (-j \omega x) f(x) \mathrm{d} x \\
& \times \int_{-T}^{T} \exp (j \omega \tilde{x}) f^{*}(\tilde{x}) \mathrm{d} x \mathrm{~d} \omega \\
& =\frac{1}{2 \pi} \int_{-T}^{T} \int_{-T}^{T}\left\{\int_{-\Omega}^{\Omega} \exp [j \omega(\tilde{x}\right. \\
& -x)] \mathrm{d} \omega\} f(x) f^{*}(\tilde{x}) \mathrm{d} x \mathrm{~d} \tilde{x} \\
& =\int_{-T}^{T} \int_{-T}^{T} K_{F, \Omega}(x, \tilde{x}) f(x) f^{*}(\tilde{x}) \mathrm{d} x \mathrm{~d} \tilde{x} \\
& =\int_{-T}^{T}\left[\sum_{n=0}^{\infty} a_{n} \int_{-T}^{T} K_{F, \Omega}(x, \tilde{x}) \psi_{n}(x) \mathrm{d} x\right] \\
& \times \sum_{m=0}^{\infty} a_{m}^{*} \psi_{m}(\tilde{x}) \mathrm{d} \tilde{x} \\
& =\int_{-T}^{T} \sum_{n=0}^{\infty} \lambda_{n} a_{n} \psi_{n}(\tilde{x}) \sum_{m=0}^{\infty} a_{m}^{*} \psi_{m}(\tilde{x}) \mathrm{d} \tilde{x} \\
& =\sum_{n=0}^{\infty} \sum_{m=0}^{\infty} \lambda_{n} a_{n} a_{m}^{*} \int_{-T}^{T} \psi_{n}(\tilde{x}) \psi_{m}(\tilde{x}) \mathrm{d} \tilde{x} \\
& =\sum_{n=0}^{\infty} \sum_{m=0}^{\infty} \lambda_{n} a_{n} a_{m}^{*} \lambda_{n} \delta_{m, n}=\sum_{n=0}^{\infty}\left|a_{n}\right|^{2} \lambda_{n}^{2},
\end{aligned}
$$

where $K_{F, \Omega}(x, \tilde{x})$ is defined in Eq. (5) and we have applied Eqs. (5), (7), and (16). Similarly,

$$
\begin{aligned}
\int_{-T}^{T}|f(x)|^{2} \mathrm{~d} x & =\int_{-T}^{T} f(x) f^{*}(x) \mathrm{d} \omega \\
& =\int_{-T n=0}^{T} \sum_{n}^{\infty} a_{n}(x) \sum_{m=0}^{\infty} a_{m}^{*} \psi_{m}(x) \mathrm{d} x \\
& =\sum_{n=0}^{\infty} \sum_{m=0}^{\infty} a_{n} a_{m}^{*} \int_{-T}^{T} \psi_{n}(x) \psi_{m}(x) \mathrm{d} x \\
& =\sum_{n=0}^{\infty} \sum_{m=0}^{\infty} a_{n} a_{m}^{*} \lambda_{n} \delta_{m, n}=\sum_{n=0}^{\infty}\left|a_{n}\right|^{2} \lambda_{n}
\end{aligned}
$$


[applying Eq. (7)]. Thus the energy ratio of $\widetilde{F}(\omega)$ to $f(t)$ is $^{1}$

$$
\begin{aligned}
\text { energy-preservation ratio } & =\frac{\int_{-\Omega}^{\Omega}|\tilde{F}(\omega)|^{2} \mathrm{~d} \omega}{\int_{-T}^{T}|f(x)|^{2} \mathrm{~d} x} \\
& =\frac{\sum_{n=0}^{\infty}\left|a_{n}\right|^{2} \lambda_{n}^{2}}{\sum_{n=0}^{\infty}\left|a_{n}\right|^{2} \lambda_{n}} .
\end{aligned}
$$

Since $\lambda_{0}>\lambda_{n}$, if $n \neq 0$, to make the energy ratio maximal, $f(x)$ should be a multiple of $\psi_{0}(x)$ :

$$
f(x)=a_{0} \psi_{0}(x)
$$

In addition to analyzing the energy-preservation problem of the fi-FT, PSWFs are also useful for finite-sized optical system analysis, laser, extrapolation, and finite-extension filter design. ${ }^{1-4,7,8}$

In this paper we generalize the theory of PSWFs and discuss how to use it to deal with the problems associated with the finite-extension fractional Fourier transform (fiFRFT), the finite-extension linear canonical transform (fiLCT), and the offset finite-extension linear canonical transform (offset fi-LCT). These finite transforms are more generalized than the fi-FT. Many finite-sized optical systems that cannot be represented by the fi-FT can be modeled by the fi-FRFT, the fi-LCT, or the offset fi-LCT. After the generalized prolate spheroidal functions (GPSWFs) corresponding to these finite transforms are derived, we can use them to analyze the properties of the more generalized finite-sized optical system.

In Section 2 we derive the GPSWFs corresponding to the fi-FRFT, the fi-LCT, and the offset fi-LCT. We consider not only the case where the interval is centered at zero but also the case where the center departs from zero. In Section 3 we derive some important properties of GPSWFs. In Section 4 we illustrate how to use GPSWFs to analyze the energy-preservation ratio, the self-imaging phenomenon, and the resonance phenomenon of the finite-sized optical system. We also consider the case in which the optical system is a cascade of several finitesized optical systems, i.e., the multiple-stage case.

\section{GENERALIZED PROLATE SPHEROIDAL FUNCTIONS FOR FINITE-EXTENSION FRFTs, LCTs, AND OFFSET LCTs}

A. Definitions of Fi-FRFT, Fi-LCT, and Offset Fi-LCT

The fractional Fourier transform (FRFT) is defined as ${ }^{9,10}$

$$
\begin{aligned}
G_{a}(u)= & O_{F}^{\alpha}[g(x)]=\left(\frac{1-j \cot \alpha}{2 \pi}\right)^{1 / 2} \\
& \times \int_{-\infty}^{\infty} \exp \left(\frac{j}{2} u^{2} \cot \alpha-j u x \csc \alpha\right. \\
& \left.+\frac{j}{2} x^{2} \cot \alpha\right) g(x) \mathrm{d} x .
\end{aligned}
$$

It is a generalization of the FT. When $\alpha=\pi / 2$, it becomes the FT. When $\alpha=-\pi / 2$, it becomes the inverse FT. The linear canonical transform (LCT) is a further generalization of FRFTs. It is defined as ${ }^{11,12}$

$$
\begin{aligned}
G_{(a, b, c, d)}(u)= & O_{F}^{(a, b, c, d)}[g(x)]=\left(\frac{1}{j 2 \pi b}\right)^{1 / 2} \\
& \times \int_{-\infty}^{\infty} \exp \left(\frac{j}{2} \frac{d}{b} u^{2}-j \frac{u}{b} x+\frac{j}{2} \frac{a}{b} x^{2}\right) \\
& \times g(x) \mathrm{d} x
\end{aligned}
$$

The FRFT is a special case of the LCT, where $\{a, b, c, d\}=\{\cos \alpha, \sin \alpha,-\sin \alpha, \cos \alpha\}$,

$$
O_{F}^{a}[g(x)]=[\exp (j \alpha)]^{1 / 2} O_{F}^{(\cos \alpha, \sin \alpha,-\sin \alpha, \cos \alpha)}[g(x)] .
$$

The Fresnel transform ${ }^{11}$ is a special case of the LCT (where $\{a, b, c, d\}=\{1, z \lambda / 2 \pi, 0,1\}$ ). The FRFT and the LCT are more flexible than the original FT. They are useful for filter design, communication, space-variant pattern recognition, phase retrieval, encryption, optical system analysis, ${ }^{12-14}$ spherical mirror-pair-system analysis, ${ }^{15}$ etc.

The LCT can be further generalized into the offset linear canonical transform (offset LCT). ${ }^{11,16,17}$ It has two extra parameters, $\tau$ and $\rho$, which represents the space and frequency offsets:

$$
\begin{aligned}
O_{F}^{(a, b, c, d, \tau, \rho)}[g(x)] & \\
= & (j 2 \pi b)^{-1 / 2} \exp (j \rho u) \int_{-\infty}^{\infty} \exp \left[\frac{j}{2} \frac{d}{b}(u-\tau)^{2}\right. \\
& \left.-j \frac{u-\tau}{b} x+\frac{j}{2} \frac{a}{b} x^{2}\right] g(x) \mathrm{d} x .
\end{aligned}
$$

The offset LCT can analyze the optical system that consists of the lens, the misaligned component, or the component whose width is the form of $\exp \left[j\left(h_{2} x^{2}+h_{1} x+h_{0}\right)\right]$.

Notice that, in Eqs. (21), (22), and (24), the space intervals are $x \in(-\infty, \infty)$. However, the infinite interval is not practical. For example, we usually use an optical system to implement the FRFT, the LCT, and the offset LCT. An infinite space interval means the sizes of the optical components are infinite. It is obviously impossible. It is more practical to use the fi-FRFT, the fi-LCT, and the offset fi-LCT to replace the FRFT, the LCT, and the offset LCT with infinite intervals. 
1. fi-FRFT:

$$
\begin{aligned}
\widetilde{G}_{a}(u)= & O_{\tilde{F}}^{a}[g(x)] \\
= & \left(\frac{1-j \cot \alpha}{2 \pi}\right)^{1 / 2} \\
& \times \int_{T_{1}}^{T_{2}} \exp \left(\frac{j}{2} u^{2} \cot \alpha-j u x \csc \alpha\right. \\
& \left.+\frac{j}{2} x^{2} \cot \alpha\right) g(x) \mathrm{d} x, \\
x \in & {\left[T_{1}, T_{2}\right], \quad u \in\left[\Omega_{1}, \Omega_{2}\right] . }
\end{aligned}
$$

In addition, Khare and George used the original PSWF together with the fractionalizations of eigenvalues to define the fi-FRFT. ${ }^{18}$ That is,

$$
\widetilde{G}_{\alpha}(u)=\int_{T^{1 / 2} \Omega^{-1 / 2}}^{T^{1 / 2} \Omega^{-1 / 2}} \hat{K}_{\alpha}(u, x) g\left(T^{1 / 2} \Omega^{-1 / 2} x\right) \mathrm{d} x,
$$

where

$$
\begin{aligned}
\hat{K}_{\alpha}(u, x)= & \sum_{n=0}^{\infty} i^{-\beta}\left(T \Omega^{-1} \lambda_{n}\right)^{-\beta} \phi_{n}\left(T^{1 / 2} \Omega^{-1 / 2} u\right) \\
& \times \phi_{n}\left(T^{1 / 2} \Omega^{-1 / 2} x\right), \quad \beta=2 \alpha \pi^{-1} .
\end{aligned}
$$

In this paper, for the consideration of optics analysis, we use Eq. (25) as the definition of the fi-FRFT.

2. fi-LCT:

$$
\begin{aligned}
& \tilde{G}_{(a, b, c, d)}(u) \\
& =O_{\tilde{F}}^{(a, b, c, d)}[g(x)] \\
& =\left(\frac{1}{j 2 \pi b}\right)^{1 / 2} \\
& \quad \times \int_{T_{1}}^{T_{2}} \exp \left(\frac{j}{2} \frac{d}{b} u^{2}-j \frac{u}{b} x+\frac{j}{2} \frac{a}{b} x^{2}\right) g(x) \mathrm{d} x, \\
& a d-b c=1, \quad x \in\left[T_{1}, T_{2}\right], \quad u \in\left[\Omega_{1}, \Omega_{2}\right] .
\end{aligned}
$$

offset fi-LCT:

$$
\begin{aligned}
& \widetilde{G}_{(a, b, c, d, \tau, \rho)}(u)= O_{\widetilde{F}}^{(a, b, c, d, \tau, \rho)}[g(x)] \\
&= \frac{\exp (j \rho u)}{(j 2 \pi b)^{1 / 2}} \int_{T_{1}}^{T_{2}} \exp \left[\frac{j d}{2 b}(u-\tau)^{2}\right. \\
&\left.-j \frac{u-\tau}{b} x+\frac{j a}{2 b} x^{2}\right] g(x) \mathrm{d} x, \\
& a d-b c=1, \quad x \in\left[T_{1}, T_{2}\right], \quad u \in\left[\Omega_{1}, \Omega_{2}\right] .
\end{aligned}
$$

\section{B. Generalized Prolate Spheroidal Functions for Fi-FRFTs and Fi-LCTs}

Here we derive the function set that can analyze the energy-preservation property of the fi-FRFT and the fiLCT. Here we consider the case where the intervals are centered at zero, i.e., in Eqs. (25) and (27),

$$
\left[T_{1}, T_{2}\right]=[-T, T], \quad\left[\Omega_{1}, \Omega_{2}\right]=[-\Omega, \Omega] .
$$

Since the fi-FRFT is a special case of the fi-LCT, in the following, we just discuss the case of the fi-LCT. The energy-preservation ratio of the fi-LCT can be calculated from

$$
R(\text { energy-pres. ratio })=\frac{\int_{-\Omega}^{\Omega}\left|\widetilde{G}_{(a, b, c, d)}(u)\right|^{2} \mathrm{~d} u}{\int_{-T}^{T}|g(x)|^{2} \mathrm{~d} x} .
$$

From Eq. (27),

$$
\begin{aligned}
\int_{-\Omega}^{\Omega}\left|\widetilde{G}_{(a, b, c, d)}(u)\right|^{2} \mathrm{~d} u \\
=\int_{-\Omega}^{\Omega} \widetilde{G}_{(a, b, c, d)}(u) \widetilde{G}_{(a, b, c, d)}^{*}(u) \mathrm{d} u \\
=\frac{1}{2 \pi|b|} \int_{-\Omega}^{\Omega} \int_{-T}^{T} \int_{-T}^{T} \exp \left(\frac{j}{2} \frac{d}{b} u^{2}-j \frac{u}{b} x+\frac{j}{2} \frac{a}{b} x^{2}\right) \\
\quad \times \exp \left(-\frac{j}{2} \frac{d}{b} u^{2}+j \frac{u}{b} \tilde{x}-\frac{j}{2} \frac{a}{b} \tilde{x}^{2}\right) g(x) g *(\tilde{x}) \mathrm{d} x \mathrm{~d} \tilde{x} \mathrm{~d} u \\
=\frac{1}{2 \pi|b|} \int_{-T}^{T} \int_{-T}^{T}\left\{\int_{-\Omega}^{\Omega} \exp \left[j \frac{u}{b}(\tilde{x}-x)\right] \mathrm{d} u\right\} \\
\quad \times \exp \left[\frac{j}{2} \frac{a}{b}\left(x^{2}-\tilde{x}^{2}\right)\right] g(x) g *(\tilde{x}) \mathrm{d} x \mathrm{~d} \tilde{x} \\
=\int_{-T}^{T} \int_{-T}^{T} \frac{\sin \left[\Omega(\tilde{x}-x)|b|^{-1}\right]}{\pi(\tilde{x}-x)} \\
\quad \times \exp \left[\frac{j}{2} \frac{a}{b}\left(x^{2}-\tilde{x}^{2}\right)\right] g(x) g *(\tilde{x}) \mathrm{d} x \mathrm{~d} \tilde{x} .
\end{aligned}
$$

Then we try to find the energy eigenfunction that satisfies the following homogeneous integral equation:

$$
\eta_{n, T, \Omega} \phi_{n, T, \Omega}(\tilde{x})=\int_{-T}^{T} K_{(a, b, c, d), \Omega}(\tilde{x}, x) \phi_{n, T, \Omega}(x) \mathrm{d} x,
$$

where

$$
\begin{aligned}
K_{(a, b, c, d), \Omega}(\tilde{x}, x)= & \frac{\sin \left[\Omega(\tilde{x}-x)|b|^{-1}\right]}{\pi(\tilde{x}-x)} \\
& \times \exp \left[\frac{j}{2} \frac{a}{b}\left(x^{2}-\tilde{x}^{2}\right)\right], \\
& x, \tilde{x} \in[-T, T] .
\end{aligned}
$$

Then we compare it with the integral kernel of the original PSWFs in Eq. (5). Since

$$
\begin{aligned}
& K_{(a, b, c, d), \Omega}(\tilde{x}, x) \\
& \quad=|b|^{-1} K_{F, \Omega}\left(|b|^{-1} \tilde{x},|b|^{-1} x\right) \exp \left[\frac{j a}{2 b}\left(x^{2}-\tilde{x}^{2}\right)\right],
\end{aligned}
$$


if $\psi_{n, T|b|^{-1}, \Omega}(x)$ is the PSWF of the original fi-FT, which satisfies

$\lambda_{n, T|b|^{-1}, \Omega} \psi_{n, T|b|^{-1}, \Omega}(\tilde{x})$

$$
=\int_{-T|b|^{-1}}^{T|b|^{-1}} K_{F, \Omega}(\tilde{x}, x) \psi_{n, T|b|^{-1}, \Omega}(x) \mathrm{d} x,
$$

then the solution of Eq. (32), i.e., the GPSWFs for the fi$\mathrm{LCT}$ and the corresponding eigenvalues are

$$
\begin{array}{r}
\phi_{n, T, \Omega}(x)=|b|^{-1 / 2} \exp \left(-\frac{j}{2} \frac{a}{b} x^{2}\right) \psi_{n, T|b|^{-1, \Omega}}\left(|b|^{-1} x\right), \\
\eta_{n, T, \Omega}=\lambda_{n, T|b|^{-1, \Omega}},
\end{array}
$$

since

$$
\begin{aligned}
& \int_{-T}^{T} K_{(a, b, c, d), \Omega}(\tilde{x}, x) \phi_{n, T, \Omega}(x) \mathrm{d} x \\
& =|b|^{-3 / 2} \exp \left[-j a \tilde{x}^{2}(2 b)^{-1}\right] \\
& \quad \times \int_{-T}^{T} K_{F, \Omega}\left(|b|^{-1} \tilde{x},|b|^{-1} x\right) \psi_{n, T|b|^{-1, \Omega}}\left(|b|^{-1} x\right) \mathrm{d} x \\
& =|b|^{-1 / 2} \exp \left[-j a \tilde{x}^{2}(2 b)^{-1}\right] \\
& \quad \times \int_{-T|b|^{-1}}^{T|b|^{-1}} K_{F, \Omega}\left(|b|^{-1} \tilde{x}, x_{1}\right) \psi_{n, T|b|^{-1, \Omega}}\left(x_{1}\right) \mathrm{d} x_{1} \\
& =\lambda_{n, T|b|^{-1, \Omega}}|b|^{-1 / 2} \exp \left(-\frac{j}{2} \frac{a}{b} \tilde{x}^{2}\right) \psi_{n, T|b|^{-1, \Omega}}\left(|b|^{-1} \tilde{x}\right) \\
& =\eta_{n, T, \Omega} \phi_{n, T, \Omega}(\tilde{x}) .
\end{aligned}
$$

As in the case of original PSWFs, all the eigenvalues $\eta_{n, T, \Omega}$ 's are real, positive, and smaller than 1 . We can sort $\phi_{n, T, \Omega}(x)$ and $\eta_{n, T, \Omega}$ such that $\eta_{m, T, \Omega}>\eta_{n, T, \Omega}$ if $m$ $>n$ :

$$
1>\eta_{0, T, \Omega}>\eta_{1, T, \Omega}>\eta_{2, T, \Omega}>\eta_{3, T, \Omega}>\cdots>0 .
$$

To compute GPSWFs numerically, we can first use the process in expressions (12)-(15) to calculate original PSWFs. Then we apply Eq. (35) to compute the GPSWF for the fi-LCT from the original PSWFs.

GPSWFs are orthogonal in the intervals of both $x$ $\in[-T, T]$ and $x \in(-\infty, \infty)$ :

$$
\begin{aligned}
\int_{-T}^{T} & \phi_{n, T, \Omega}(x) \phi_{m, T, \Omega}^{*}(x) \mathrm{d} x \\
& =|b|^{-1} \int_{-T}^{T} \psi_{n, T|b|^{-1, \Omega}}\left(|b|^{-1} x\right) \psi_{m, T|b|^{-1, \Omega}}^{*}\left(|b|^{-1} x\right) \mathrm{d} x \\
& =\int_{-T|b|^{-1}}^{T|b|^{-1}} \psi_{n, T|b|^{-1, \Omega}}\left(x_{1}\right) \psi_{m, T|b|^{-1, \Omega}}^{*}\left(x_{1}\right) \mathrm{d} x_{1} \\
& =\lambda_{n, T|b|^{-1, \Omega}} \delta_{m, n}=\eta_{n, T, \Omega} \delta_{m, n},
\end{aligned}
$$

$$
\begin{aligned}
\int_{-\infty}^{\infty} & \phi_{n, T, \Omega}(x) \phi_{m, T, \Omega}^{*}(x) \mathrm{d} x \\
& =|b|^{-1} \int_{-\infty}^{\infty} \psi_{n, T|b|^{-1, \Omega}}\left(|b|^{-1} x\right) \psi_{m, T|b|^{-1, \Omega}}^{*}\left(|b|^{-1} x\right) \mathrm{d} x \\
& =\int_{-\infty}^{\infty} \psi_{n, T|b|^{-1, \Omega}}\left(x_{1}\right) \psi_{m, T|b|^{-1, \Omega}}^{*}\left(x_{1}\right) \mathrm{d} x_{1}=\delta_{m, n} .
\end{aligned}
$$

Moreover, since the original PSWFs $\left\{\psi_{0}(x), \psi_{1}(x), \psi_{2}(x), \psi_{3}(x) \ldots\right\} \quad$ are complete, so $\left\{\phi_{0}(x), \phi_{1}(x), \phi_{2}(x), \phi_{3}(x) \ldots\right\}$ is also complete. Since $\left\{\phi_{n}(x) \mid n \in Z^{+}\right\}$forms an orthogonal and complete function set, any function can be expressed as a linear combination of $\phi_{0}(x), \phi_{1}(x), \phi_{2}(x), \phi_{3}(x) \ldots$ :

$$
\begin{aligned}
g(x) & =\sum_{n=0}^{\infty} \sigma_{n} \phi_{n}(x), \quad x \in[-T, T], \\
\sigma_{n} & =\eta_{n}^{-1} \int_{-T}^{T} g(x) \phi_{n}^{*}(x) \mathrm{d} x .
\end{aligned}
$$

Thus

$$
\begin{aligned}
\int_{-T}^{T}|g(x)|^{2} \mathrm{~d} x & =\sum_{n=0}^{\infty} \sum_{m=0}^{\infty} \sigma_{n} \sigma_{m}^{*} \int_{-T}^{T} \phi_{n}(x) \phi_{m}^{*}(x) \mathrm{d} x \\
& =\sum_{n=0}^{\infty}\left|\sigma_{n}\right|^{2} \eta_{n} .
\end{aligned}
$$

Moreover, from Eq. (31),

$$
\begin{aligned}
\int_{-\Omega}^{\Omega}\left|\widetilde{G}_{(a, b, c, d)}(u)\right|^{2} \mathrm{~d} u \\
=\int_{-T}^{T} \int_{-T}^{T} K_{(a, b, c, d), \Omega}(\tilde{x}, x) g(x) g^{*}(\tilde{x}) \mathrm{d} x \mathrm{~d} \tilde{x} \\
=\int_{-T}^{T}\left[\int_{-T}^{T} K_{(a, b, c, d), \Omega}(\tilde{x}, x) \sum_{n=0}^{\infty} \sigma_{n} \phi_{n}(x) \mathrm{d} x\right] \\
\quad \times \sum_{m=0}^{\infty} \sigma_{m}^{*} \phi_{m}^{*}(\tilde{x}) \mathrm{d} \tilde{x} \\
=\int_{-T n=0}^{T} \sum_{n}^{\infty} \sigma_{n} \eta_{n} \phi_{n}(\tilde{x}) \sum_{m=0}^{\infty} \sigma_{m}^{*} \phi_{m}^{*}(\tilde{x}) \mathrm{d} \tilde{x} \\
=\sum_{n=0}^{\infty} \sum_{m=0}^{\infty} \sigma_{n} \sigma_{m}^{*} \eta_{n} \int_{-T}^{T} \phi_{n}(\tilde{x}) \phi_{m}^{*}(\tilde{x}) \mathrm{d} \tilde{x} \\
=\sum_{n=0}^{\infty}\left|\sigma_{n}\right|^{2} \eta_{n}^{2} ;
\end{aligned}
$$

therefore the energy-preservation ratio $R$ for the fi-LCT is

$$
R=\frac{\int_{-\Omega}^{\Omega}\left|\widetilde{G}_{(a, b, c, d)}(u)\right|^{2} \mathrm{~d} u}{\int_{-T}^{T}|g(x)|^{2} \mathrm{~d} x}=\frac{\sum_{n=0}^{\infty}\left|\sigma_{n}\right|^{2} \eta_{n}^{2}}{\sum_{n=0}^{\infty}\left|\sigma_{n}\right|^{2} \eta_{n}} .
$$

Thus we can use GPSWFs to analyze the energypreservation property of the fi-LCT. Notice that the 
maximal and the minimal values of the energypreservation ratio are

$$
\begin{aligned}
& \operatorname{Max}(R)=\eta_{0} \approx 1, \quad g(x)=\sigma_{0} \phi_{0}(x), \\
& \min (R)=\eta_{n} \approx 0, \quad g(x)=\sigma_{n} \phi_{n}(x), \quad n \rightarrow \infty .
\end{aligned}
$$

From Eq. (35) it can be seen that, although the LCT has four parameters $\{a, b, c, d\}$, only two of them ( $a$ and $b$ ) affect the form of GPSWFs. We illustrate this in property 3 of Section 3.

In the above, we discuss the case of the fi-LCT. For the case of the fi-FRFT, we can apply the fact that the fi-FRFT is a special case of the fi-LCT, where $\{a, b, c, d\}$ $=\{\cos \alpha, \sin \alpha,-\sin \alpha, \cos \alpha\}$ [see Eq. (23)]. Thus the GPSWFs for the fi-FRFT are

$$
\begin{aligned}
\phi_{n, T, \Omega}(x)= & |\csc \alpha|^{1 / 2} \\
& \times \exp \left(-\frac{j}{2} x^{2} \cot \alpha\right) \psi_{n, T|\csc \alpha|, \Omega}(|\csc \alpha| x) \\
\eta_{n, T, \Omega}= & \lambda_{n, T|\csc \alpha|, \Omega}
\end{aligned}
$$

where $\psi_{n, T|\csc \alpha|, \Omega}(x)$ is the original PSWF that satisfies

$$
\begin{aligned}
& \lambda_{n, T|\csc \alpha|, \Omega} \psi_{n, T|\csc \alpha|, \Omega}(\tilde{x}) \\
&=\int_{-T|\csc \alpha|}^{T|\csc \alpha|} \frac{\sin [\Omega(\tilde{x}-x)]}{\pi(\tilde{x}-x)} \psi_{n, T|\csc \alpha|, \Omega}(x) \mathrm{d} x .
\end{aligned}
$$

The GPSWFs of the fi-FRFT are orthogonal and complete. After the fi-FRFT is done, the power-preservation ratio can be calculated from

$$
\begin{gathered}
R=\frac{\int_{-\Omega}^{\Omega}\left|\widetilde{G}_{\alpha}(u)\right|^{2} \mathrm{~d} u}{\int_{-T}^{T}|g(x)|^{2} \mathrm{~d} x}=\frac{\sum_{n=0}^{\infty}\left|\sigma_{n}\right|^{2} \eta_{n}^{2}}{\sum_{n=0}^{\infty}\left|\sigma_{n}\right|^{2} \eta_{n}}, \\
\sigma_{n}=\eta_{n}^{-1} \int_{-T}^{T} g(x) \phi_{n}^{*}(x) \mathrm{d} x .
\end{gathered}
$$

\section{Generalized Prolate Spheroidal Functions for Offset Fi-LCTs}

In this subsection we further generalize the result in Subsection 2.B into the case of the finite-sized offset fi-LCT. Here we consider only the case where the intervals are centered at zero, i.e., $x \in[-T, T]$ and $\omega \in[-\Omega, \Omega]$. Following a process similar to that in Eqs. (30) and (31), we find that the GPSWFs for the offset fi-LCT should satisfy the following homogeneous integral equation:

$$
\eta_{n, T, \Omega} \phi_{n, T, \Omega}(\tilde{x})=\int_{-T}^{T} K_{(a, b, c, d, \tau, \rho), \Omega}(\tilde{x}, x) \phi_{n, T, \Omega}(x) \mathrm{d} x,
$$

where
$K_{(a, b, c, d, \tau, \rho), \Omega}(\tilde{x}, x)$

$$
\begin{aligned}
& =\frac{\sin \left[\Omega(\tilde{x}-x)|b|^{-1}\right]}{\pi(\tilde{x}-x)} \\
& \times \exp \left\{j\left[\frac{\tau}{b}(x-\tilde{x})+\frac{a}{2 b}\left(x^{2}-\tilde{x}^{2}\right)\right]\right\}, \\
& x, \tilde{x} \in[-T, T] .
\end{aligned}
$$

Comparing Eq. (47) with Eq. (32), we obtain

$$
K_{(a, b, c, d, \tau, \rho), \Omega}(\tilde{x}, x)=\exp \left[\frac{j \tau}{b}(x-\tilde{x})\right] K_{(a, b, c, d), \Omega}(\tilde{x}, x) .
$$

Thus it is easy to show that

$$
\phi_{n, T, \Omega}(x)=\exp (-j \tau x / b) \phi_{n, T, \Omega}^{(\mathrm{fi}-\mathrm{LCT})}(x),
$$

where $\phi_{n, T, \Omega}^{(\mathrm{fi}-\mathrm{LCT})}(x)$ is the GPSWF of the fi-LCT [see Eq. (35)]. Thus the GPSWFs for the offset fi-LCT and the corresponding eigenvalue are

$$
\begin{aligned}
\phi_{n, T, \Omega}(x)= & |b|^{-1 / 2} \exp \left[-j\left(\frac{a}{2 b} x^{2}+\frac{\tau}{b} x\right)\right] \\
& \times \psi_{n, T|b|^{-1}, \Omega}\left(|b|^{-1} x\right), \\
\eta_{n, T, \Omega}= & \lambda_{n, T|b|^{-1, \Omega}},
\end{aligned}
$$

where $\psi_{n, T|b|^{-1}, \Omega}(x)$ and $\lambda_{n, T|b|^{-1}, \Omega}$ are the original PSWF and the corresponding eigenvalue that satisfy Eq. (34). Notice that the GPSWFs for the offset fi-LCT are just the modulation of that of the fi-LCT. The offset fi-LCT has six parameters; however, only three of them $(a, b$, and $\tau)$ affect the form of GPSWFs. The GPSWFs of the offset fi-LCT also satisfy the orthogonal properties in Eq. (38). They form a complete orthogonal functions set in the interval of $x \in[-T, T]$. We can also use the way used in Eq. (42) to analyze the energy-preservation ratio of the offset fi-LCT.

\section{Cases of Nonsymmetric Intervals}

In Subsections 2.B and 2.C we discussed how to use GPSWFs to analyze the energy-preservation ratio for the fi-FRFT, the fi-LCT, and the offset fi-LCT. We limited the space interval and the frequency interval to be symmetric about zero (i.e., $x \in[-T, T]$ and $u \in[-\Omega, \Omega]$ ). However, in practice, it is very often that the intervals are not symmetric. In this subsection we discuss the cases in which the space and frequency intervals are not centered at zero.

Notice that the nonsymmetrical intervals case is equivalent to the symmetric one together with the shifts in the time and the frequency domains. Thus assume that $\hat{G}(u)$ is the offset fi-LCT of $g(x)$ whose intervals are not centered at zero:

$$
\hat{G}(u)=\int_{T_{1}}^{T_{2}} \Gamma_{(a, b, c, d, \tau, \rho)}(u, x) g(x) \mathrm{d} x,
$$

where 


$$
\begin{aligned}
& \Gamma_{(a, b, c, d, \tau, \rho)}(u, x) \\
& \quad=\frac{\exp (j \rho u)}{(j 2 \pi b)^{1 / 2}} \exp \left[\frac{j d}{2 b}(u-\tau)^{2}-j \frac{u-\tau}{b} x+\frac{j a}{2 b} x^{2}\right], \\
& x \in\left[T_{1}, T_{2}\right], \quad u \in\left[\Omega_{1}, \Omega_{2}\right], \quad T_{1} \neq-T_{2}, \\
& \Omega_{1} \neq-\Omega_{2} .
\end{aligned}
$$

We can shift $g(x)$ and $\hat{G}(u)$ and convert the intervals into symmetrical ones:

$$
\begin{array}{ll}
h(x)=g\left(x+T_{0}\right) & \text { for } h(x), \quad x \in\left[-T_{3}, T_{3}\right], \\
\hat{H}(u)=\hat{G}\left(u+\Omega_{0}\right) & \text { for } \hat{H}(u), \quad u \in\left[-\Omega_{3}, \Omega_{3}\right],
\end{array}
$$

where

$$
\begin{array}{ll}
T_{0}=2^{-1}\left(T_{1}+T_{2}\right), & \Omega_{0}=2^{-1}\left(\Omega_{1}+\Omega_{2}\right), \\
T_{3}=2^{-1}\left(T_{2}-T_{1}\right), & \Omega_{3}=2^{-1}\left(\Omega_{2}-\Omega_{1}\right) .
\end{array}
$$

Then, from Eqs. (51) and (53), we can show that the relation between $h(x)$ and $\hat{H}(u)$ is

$$
\hat{H}(u)=\int_{-T_{3}}^{T_{3}} \Gamma_{(a, b, c, d, \tau, \rho)}\left(u+\Omega_{0}, x+T_{0}\right) h(x) \mathrm{d} x .
$$

From Eq. (51), after some calculation, we can prove that

$$
\begin{aligned}
\Gamma_{(a, b, c, d, \tau, \rho)}\left(u+\Omega_{0}, x\right. & \left.+T_{0}\right) \\
& =\exp (j \varphi) \Gamma_{\left(a, b, c, d, \tau_{1}, \rho_{1}\right)}(u, x),
\end{aligned}
$$

where $\varphi$ is some constant phase and

$$
\begin{gathered}
\tau_{1}=\tau+a T_{0}-\Omega_{0}, \\
\rho_{1}=\rho+b^{-1}(a d-1) T_{0}=\rho+c T_{0} .
\end{gathered}
$$

Thus Eq. (55) can be rewritten as

$$
\begin{aligned}
\hat{H}(u) & =\exp (j \varphi) \int_{-T_{3}}^{T_{3}} \Gamma_{\left(a, b, c, d, \tau_{1}, \rho_{1}\right)}(u, x) h(x) \mathrm{d} x, \\
x & \in\left[-T_{3}, T_{3}\right], \quad u \in\left[-\Omega_{3}, \Omega_{3}\right] .
\end{aligned}
$$

That is, the relation between $h(x)$ and $\hat{H}(u)$ can be expressed as the offset fi-LCT with parameters $\left\{a, b, c, d, \tau_{1}, \rho_{1}\right\}$, and the intervals of the offset fi-LCT are centered at zero. From Eq. (50) in Subsection 2.C, we know that the GPSWFs corresponding to the offset fi-LCT in Eq. (58) are

$$
\begin{aligned}
& \phi_{n, T_{3}, \Omega_{3}}(x)=|b|^{-1 / 2} \exp \left[-j\left(\frac{a}{2 b} x^{2}+\frac{\tau+a T_{0}-\Omega_{0}}{b} x\right)\right] \\
& \times \psi_{n, T_{3}|b|^{-1}, \Omega_{3}}\left(|b|^{-1} x\right) .
\end{aligned}
$$

Since $g(x)=h\left(x-T_{0}\right)$, we can conclude that the GPSWFs for the offset fi-LCT whose intervals are not centered at zero and the corresponding eigenvalue are (we removed the constant phase term for simplification)

$$
\begin{aligned}
\phi_{n, T_{1}, T_{2}, \Omega_{1}, \Omega_{2}}(x)= & \phi_{n, T_{3}, \Omega_{3}}\left(x-T_{0}\right) \\
= & |b|^{-1 / 2} \exp \left(-\frac{j a}{2 b} x^{2}+j \frac{\Omega_{0}-\tau}{b} x\right) \\
& \times \psi_{n, T_{3}|b|^{-1}, \Omega_{3}}\left(\frac{x-T_{0}}{|b|}\right), \\
\eta_{n, T_{1}, T_{2}, \Omega_{1}, \Omega_{2}}= & \lambda_{n, T_{3}|b|^{-1}, \Omega_{3}},
\end{aligned}
$$

where $T_{0}=2^{-1}\left(T_{1}+T_{2}\right), \quad \Omega_{0}=2^{-1}\left(\Omega_{1}+\Omega_{2}\right), \quad T_{3}$ $=2^{-1}\left(T_{2}-T_{1}\right), \quad \Omega_{3}=2^{-1}\left(\Omega_{2}-\Omega_{1}\right), \quad$ and $\psi_{n, T_{3}|b|^{-1}, \Omega_{3}}(x)$ is the original PSWF, which satisfies Eq.

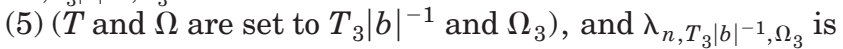
its corresponding eigenvalue.

The GPSWFs in Eq. (60) are complete and orthogonal:

$$
\begin{aligned}
& \int_{T_{1}}^{T_{2}} \phi_{n}(x) \phi_{m}^{*}(x) \mathrm{d} x=\eta_{n} \delta_{m, n}, \\
& \int_{-\infty}^{\infty} \phi_{n}(x) \phi_{m}^{*}(x) \mathrm{d} x=\delta_{m, n} .
\end{aligned}
$$

The energy-preservation ratio for the offset fi-LCT can be calculated from

$$
\begin{aligned}
& R=\frac{\int_{\Omega_{1}}^{\Omega_{2}}\left|\widetilde{G}_{(a, b, c, d)}(u)\right|^{2} \mathrm{~d} u}{\int_{T_{1}}^{T_{2}}|g(x)|^{2} \mathrm{~d} x}=\frac{\sum_{n=0}^{\infty}\left|\sigma_{n}\right|^{2} \eta_{n}^{2}}{\sum_{n=0}^{\infty}\left|\sigma_{n}\right|^{2} \eta_{n}} \\
& \sigma_{n}=\frac{\int_{T_{1}}^{T_{2}} g(x) \phi_{n}^{*}(x) \mathrm{d} x}{\eta_{n}} .
\end{aligned}
$$

\section{PROPERTIES}

In Section 2 we described that the GPSWFs for the fiFEFT, the fi-LCT, and the offset fi-LCT are complete and orthogonal. In this section we introduce other important properties of the GPSWFs.

\section{Symmetry property}

$$
\begin{gathered}
\phi_{n, T_{1}, T_{2}, \Omega_{1}, \Omega_{2}}\left(T_{0}+x\right)=(-1)^{n} \phi_{n, T_{1}, T_{2}, \Omega_{1}, \Omega_{2}}\left(T_{0}-x\right), \\
T_{0}=2^{-1}\left(T_{1}+T_{2}\right) .
\end{gathered}
$$

2. Space-frequency bandwidth product property

It is known that, for the original PSWF, if the product of the space interval and the frequency interval is fixed, when we change the space interval, PSWFs are intrinsically unchanged except for scaling:

$$
\psi_{n, \sigma T, \Omega \sigma^{-1}}(x)=\sigma^{-1 / 2} \psi_{n, T, \Omega}\left(\sigma^{-1} x\right) .
$$

This space-frequency bandwidth product property can also be applied to GPSWFs with some modifications.

The GPSWFs for the offset fi-LCT with intervals $x$ $\in\left[T_{1}, T_{2}\right]$ and $\omega \in\left[\Omega_{1}, \Omega_{2}\right]$ are shown in Eq. (60). Assume that now the intervals are changed into 


$$
\begin{aligned}
& x \in\left[T_{4}, T_{5}\right]=\left[T_{0}-\sigma T_{3}, T_{0}+\sigma T_{3}\right], \\
& \omega \in\left[\Omega_{4}, \Omega_{5}\right]=\left[\Omega_{0}-\sigma^{-1} \Omega_{3}, \Omega_{0}+\sigma^{-1} \Omega_{3}\right],
\end{aligned}
$$

where $T_{0}=2^{-1}\left(T_{1}+T_{2}\right), \quad \Omega_{0}=2^{-1}\left(\Omega_{1}+\Omega_{2}\right), \quad T_{3}$ $=2^{-1}\left(T_{2}-T_{1}\right)$, and $\Omega_{3}=2^{-1}\left(\Omega_{2}-\Omega_{1}\right)$. Then, from Eq. (60),

$$
\begin{aligned}
& \phi_{n, T_{4}, T_{5}, \Omega_{4}, \Omega_{5}}(x)=|b|^{-1 / 2} \exp \left(-\frac{j a}{2 b} x^{2}+j \frac{\Omega_{0}-\tau}{b} x\right) \\
& \times \psi_{n, \sigma T_{3}|b|^{-1}, \Omega_{3} \sigma^{-1}}\left(\frac{x-T_{0}}{|b|}\right),
\end{aligned}
$$

[applying Eq. (65)]

$$
\begin{aligned}
& =|\sigma b|^{-1 / 2} \exp \left(-\frac{j a}{2 b} x^{2}+j \frac{\Omega_{0}-\tau}{b} x\right) \\
& \times \psi_{n, T_{3}|b|^{-1}, \Omega_{3}}\left(\frac{x-T_{0}}{\sigma|b|}\right), \\
& \left|\phi_{n, T_{4}, T_{5}, \Omega_{4}, \Omega_{5}}\left(x+T_{0}\right)\right| \\
& =\left|\sigma^{-1 / 2} \phi_{n, T_{1}, T_{2}, \Omega_{1}, \Omega_{2}}\left(\frac{x+T_{0}}{\sigma}\right)\right| .
\end{aligned}
$$

That is, if the product of the space and frequency intervals is fixed, when we change the space interval, the amplitudes of GPSWFs are almost unchanged except for some scaling.

From Eq. (60), the amplitude of $\phi_{n, T_{1}, T_{2}, \Omega_{1}, \Omega_{2}}(x)$ is determined by $\psi_{n, T_{3}|b|^{-1}, \Omega_{3}}(x)$, which is the original PSWF. Moreover, when the difference of scaling is ignored, $\psi_{n, T_{3}|b|^{-1}, \Omega_{3}}(x)$ is determined by the product of $T_{3}|b|^{-1}$ and $\Omega_{3}$. Thus only the following product really affects the amplitude of GPSWFs:

$$
P=T_{3} \Omega_{3}|b|^{-1}=\left(T_{2}-T_{1}\right)\left(\Omega_{2}-\Omega_{1}\right)(4|b|)^{-1} .
$$

3. Effects of the parameters

Although the general form of the offset fi-LCT [see Eq. (28)] with nonsymmetric intervals has ten parameters $\left\{a, b, c, d, \tau, \rho, T_{1}, T_{2}, \Omega_{1}, \Omega_{2}\right\}$, notice that, from Eq. (60) and property 2 ,

(a) Only the product $P$ in Eq. (69) really affects the amplitude.

(b) The parameter $b$ affects the scale.

(c) The ratio of $a b^{-1}$ affects the chirp term.

(d) $T_{0}=2^{-1}\left(T_{1}+T_{2}\right)$ affects the amount of space shift.

(e) $\Omega_{0}=2^{-1}\left(\Omega_{1}+\Omega_{2}\right)$ and $\tau$ affect the modulation.

(f) The ratio of $T_{1}-T_{2}$ to $\Omega_{1}-\Omega_{2}$ affects the scaling. It can be realized from Eqs. (66)-(68).

(g) The parameters $c, d$, and $\rho$, in fact, have no effect. The parameter $c$ has no effect, since it is dependent on $a$, $b$, and $d(a d-b c=1)$. The parameters $d$ and $\rho$ have no effect because they are related to only the phase term in the frequency domain. They will be canceled during the process of deriving the integral kernel of GPSWFs. For example, for the case of the fi-LCT, the parameter $d$ is canceled in Eq. (31a).

4. Quasi-eigenfunction property

The fi-FT of original PSWFs are the eigenfunctions of the fi-FT when $T=\Omega$ :

$j^{-n} \lambda_{n, T, T}^{1 / 2} \psi_{n, T, T}(\omega)=(2 \pi)^{-1 / 2} \int_{-T}^{T} \exp (-j \omega x) \psi_{n, T, T}(x) \mathrm{d} x$.

If $T \neq \Omega$, then

$j^{-n}\left(\lambda_{n, T, \Omega} T \Omega^{-1}\right)^{1 / 2} \psi_{n, T, \Omega}\left(T \Omega^{-1} \omega\right)$

$$
=(2 \pi)^{-1 / 2} \int_{-T}^{T} \exp (-j \omega x) \psi_{n, T, \Omega}(x) \mathrm{d} x .
$$

We can use Eqs. (70) and (71) to derive the transformed result of the fi-LCT for GPSWFs. From Eq. (28),

$$
\begin{aligned}
O_{\widetilde{F}}^{(a, b, c, d, \tau, \rho)}\left[\phi_{n, T_{1}, T_{2}, \Omega_{1}, \Omega_{2}}(x)\right] \\
=\frac{\exp (j \rho u)}{(j 2 \pi b)^{1 / 2}} \int_{T_{1}}^{T_{2}} \exp \left[\frac{j d}{2 b}(u-\tau)^{2}-j \frac{u-\tau}{b} x\right. \\
\left.+\frac{j a}{2 b} x^{2}\right] \phi_{n, T_{1}, T_{2}, \Omega_{1}, \Omega_{2}}(x) \mathrm{d} x .
\end{aligned}
$$

We then apply Eq. (60) and obtain

$$
\begin{aligned}
& O_{\widetilde{F}}^{(a, b, c, d, \tau, \rho)}\left[\phi_{n, T_{1}, T_{2}, \Omega_{1}, \Omega_{2}}(x)\right] \\
& =\frac{\exp (j \rho u)}{(j 2 \pi b|b|)^{1 / 2}} \int_{T_{1}}^{T_{2}} \exp \left[\frac{j d}{2 b}(u-\tau)^{2}\right. \\
& \left.-j \frac{u-\Omega_{0}}{b} x\right] \psi_{n, T_{3} /|b|, \Omega_{3}}\left(\frac{x-T_{0}}{|b|}\right) \mathrm{d} x \\
& =\frac{\exp \left[j \rho u-j b^{-1}\left(u-\Omega_{0}\right) T_{0}\right]}{[j 2 \pi \operatorname{sgn}(b)]^{1 / 2}} \\
& \times \exp \left[\frac{j d}{2 b}(u-\tau)^{2}\right] \int_{-T_{3}|b|^{-1}}^{T_{3}|b|^{-1}} \exp \left[-j \frac{u-\Omega_{0}}{\operatorname{sgn}(b)} x_{1}\right] \\
& \times \psi_{n, T_{3}|b|^{-1, \Omega_{3}}}\left(x_{1}\right) \mathrm{d} x_{1} \\
& =j^{-n-1 / 2}\left(\frac{\lambda_{n, T_{3}|b|^{-1, \Omega_{3}} T_{3}}}{b \Omega_{3}}\right)^{1 / 2} \exp \left[j \frac{\Omega_{0}-u}{b} T_{0}+j \rho u\right. \\
& \left.+\frac{j d}{2 b}(u-\tau)^{2}\right] \psi_{n, T_{3}|b|^{-1}, \Omega_{3}}\left[\frac{T_{3}\left(u-\Omega_{0}\right)}{b \Omega_{3}}\right] \\
& =j^{(-n-1 / 2) \operatorname{sgn}(b)}\left(\frac{\lambda_{n, T_{3}|b|^{-1}, \Omega_{3} T_{3}}}{|b| \Omega_{3}}\right)^{1 / 2} \exp \left[j \frac{\Omega_{0}-u}{b} T_{0}\right. \\
& \left.+j \rho u+\frac{j d}{2 b}(u-\tau)^{2}\right] \psi_{n, T_{3}|b|^{-1}, \Omega_{3}}\left[\frac{T_{3}\left(u-\Omega_{0}\right)}{|b| \Omega_{3}}\right] .
\end{aligned}
$$


Here we use the fact that $\psi_{n, T, \Omega}(x)=(-1)^{n} \psi_{n, T, \Omega}(-x)$. Then, substituting Eq. (60) into Eq. (72), we obtain

$$
\begin{aligned}
O_{\widetilde{F}}^{(a, b, c, d, \tau, \rho)}\left[\phi_{n, T_{1}, T_{2}, \Omega_{1}, \Omega_{2}}(x)\right] & \\
= & \mu_{n} k_{1}^{-1 / 2} \exp \left(j k_{3} u^{2}+j k_{4} u\right) \phi_{n, T_{1}, T_{2}, \Omega_{1}, \Omega_{2}} \\
& \times\left(\frac{u-k_{2}}{k_{1}}\right),
\end{aligned}
$$

where

$$
\begin{aligned}
k_{1}= & \Omega_{3} T_{3}^{-1}, \quad k_{2}=\Omega_{0}-\Omega_{3} T_{0} T_{3}^{-1}, \\
k_{3}= & \frac{d}{2 b}+\frac{a T_{3}^{2}}{2 b \Omega_{3}^{2}}, \quad k_{4}=\rho-\frac{d}{b} \tau-\frac{T_{0}}{b} \\
& -\frac{T_{3}}{b \Omega_{3}}\left[\Omega_{0}\left(\frac{a T_{3}}{\Omega_{3}}+1\right)-\tau-a T_{0}\right], \\
\mu_{n}= & \left.j^{(-n-1 / 2) \operatorname{sgn}(b)} \eta_{n, T_{1}, T_{2}, \Omega_{1}, \Omega_{2}}^{1 / 2}\right) \\
& \times \exp \left\{\frac { j } { b } \left[\frac{d}{2} \tau^{2}+\Omega_{0} T_{0}+\frac{a}{2} \frac{k_{2}^{2}}{k_{1}^{2}}\right.\right. \\
& \left.\left.+\frac{k_{2}}{k_{1}}\left(\Omega_{0}-\tau\right)\right]\right\},
\end{aligned}
$$

$T_{0}=2^{-1}\left(T_{1}+T_{2}\right), \quad \Omega_{0}=2^{-1}\left(\Omega_{1}+\Omega_{2}\right), T_{3}=2^{-1}\left(T_{2}\right.$ - $\left.T_{1}\right)$, and $\Omega_{3}=2^{-1}\left(\Omega_{2}-\Omega_{1}\right)$. GPSWFs are not usually the eigenfunctions of the offset fi-LCT. However, from Eq. (73), we can see that, when we do the offset fiLCT for GPSWFs, the input and the output are just different in scaling, time shifting, chirp multiplication, and modulation.

In discussing the self-imaging phenomenon (see Subsection 4.C), all the above four terms can be ignored. Thus GPSWFs can be viewed as the quasi eigenfunctions of the offset fi-LCT and its special cases (including the fiFRFT and the fi-LCT).

Specifically, when

$$
\begin{aligned}
T_{1}=\Omega_{1}, & \left.T_{2}=\Omega_{2} \text { (i.e., } T_{0}=\Omega_{0}, \quad T_{3}=\Omega_{3}\right), \\
d=-a, & \rho=b^{-1}\left[(d-1) \tau+2 T_{0}\right],
\end{aligned}
$$

then, in Eqs. (73) and (74), $k_{1}=1$ and $k_{2}=k_{3}=k_{4}$ $=0$. In this case, GPSWFs are the eigenfunctions of the offset fi-LCT.

\section{FINITE-SIZED EXTENSION OPTICAL SYSTEM ANALYSIS}

\section{A. One-Stage Case}

The most common application of GPSWFs is analysis of the status of energy preservation of the optical system. It has been discussed that the FRFT, the LCT, and the offset LCT are useful for optical system analysis. ${ }^{9-17}$ However, the space interval and the frequency interval of these operations are infinite. In contrast, the size of the optical system is actually finite. Using them to analyze the optical system may cause some errors. It is more practical to use the fi-FRFT, the fi-LCT, and the offset fiLCT to analyze the optical system. Since the size of the optical system is always finite, after propagation through the system, some of the energy of light may be lost. Using GPSWFs, we can estimate the ratio of the energy that can be preserved. We can also find the condition under which the dispersed energy is minimal.

We give an example in Fig. 1. There are two media and one free space. Assume that the two media are of finite size and their thickness functions are of the quadratic form:

Medium 1. Thickness: $h_{0}+h_{1} x+h_{2} y+h_{3} x^{2}$

$$
+h_{4} y^{2}
$$

extension: $\quad x \in\left[-B_{1}, B_{1}\right]$,

$$
y \in\left[-P_{1}, P_{1}\right],
$$

Medium 2. Thickness: $s_{0}+s_{1} u+s_{2} v+s_{3} u^{2}$

$$
+s_{4} v^{2}
$$

$$
\begin{aligned}
\text { extension: } & u \in\left[-B_{2}, B_{2}\right], \\
& v \in\left[-P_{2}, P_{2}\right],
\end{aligned}
$$

Free space. Length: $z$.

The parameters of thickness and extension for a medium are illustrated in Fig. 2. The effect of the medium with thickness function $p x^{2}+q x+r$ is equivalent to the offset LCT with parameters $\{a, b, c, d, \tau, \rho\}$ $=\{1,0,2 k(n-1) p, 1,0, k(n-1) q\}$, where $k=2 \pi / \lambda$ and $n$ is the refractive index of the medium. The effect of the free space with length $z$ is equivalent to the offset LCT with parameters $\{a, b, c, d, \tau, \rho\}$ $=\{1, z / k, 0,1,0,0\}$ (i.e., the Fresnel transform). If we use $g_{i}(x, y)$ and $g_{o}(u, v)$ to represent the light distributions at the input and the output, respectively, then, from the additivity property of the offset LCT, ${ }^{16,17}$ we find that the whole system can be expressed as a special case of the offset fi-LCT:

$$
\begin{aligned}
g_{o}(u, v)= & \exp (j \epsilon) O_{F_{y}}^{\left(a_{1}, b_{1}, c_{1}, d_{1}, \tau_{1}, \rho_{1}\right)}\left\{O_{F_{x}}^{(a, b, c, d, \tau, \rho)}\right. \\
& \left.\times\left[g_{i}(x, y)\right]\right\}
\end{aligned}
$$

where $O_{F_{x}}$ and $O_{F_{y}}$ mean the one-dimensional offset fiLCT along the $x$ axis and $y$ axis, respectively; $x$ $\in\left[-B, B_{1}\right] ; \quad y \in\left[-P_{1}, P_{1}\right] ; \quad u \in\left[-B_{2}, B_{2}\right] ; \quad v$ $\in\left[-P_{2} ; P_{2}\right]$; and

$$
\begin{aligned}
{\left[\begin{array}{ll}
a & b \\
c & d
\end{array}\right] } & =\left[\begin{array}{cc}
1+2 z(n-1) h_{3} & z / k \\
2 k(n-1)\left(h_{3}+s_{3}\right)+\zeta & 1+2 z(n-1) s_{3}
\end{array}\right], \\
\left(\begin{array}{l}
\tau \\
\rho
\end{array}\right) & =\left[\begin{array}{c}
z(n-1) h_{1} \\
k(n-1)\left(h_{1}+s_{1}\right)+\kappa
\end{array}\right],
\end{aligned}
$$




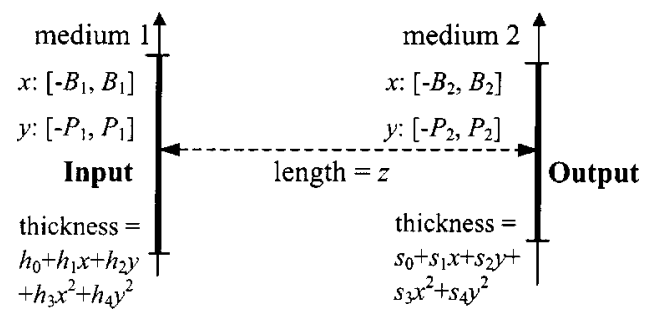

Fig. 1. Finite-sized optical system consisting of two media and one free space.

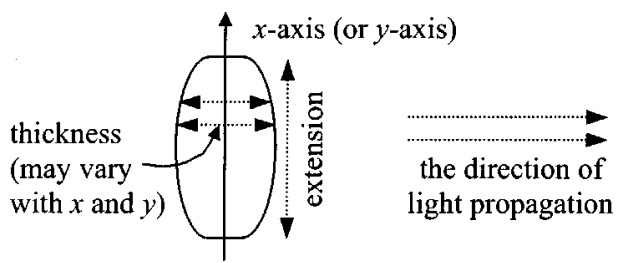

Fig. 2. Thickness and the extension of a medium.

$$
\begin{aligned}
{\left[\begin{array}{ll}
a_{1} & b_{1} \\
c_{1} & d_{1}
\end{array}\right] } & \\
& =\left[\begin{array}{cc}
1+2 z(n-1) h_{4} & z / k \\
2 k(n-1)\left(h_{4}+s_{4}\right)+\zeta_{1} & 1+2 z(n-1) s_{4}
\end{array}\right], \\
\left(\begin{array}{c}
\tau_{1} \\
\rho_{1}
\end{array}\right) & =\left[\begin{array}{c}
z(n-1) h_{2} \\
k(n-1)\left(h_{2}+s_{2}\right)+\kappa_{1}
\end{array}\right], \\
\varsigma & =4 z k(n-1)^{2} h_{3} s_{3}, \quad \varsigma_{1}=4 z k(n-1)^{2} h_{4} s_{4}, \\
\kappa & =2 k z(n-1)^{2} h_{1} s_{3}, \quad \kappa_{1}=2 k z(n-1)^{2} h_{2} s_{4}, \\
k & =2 \pi / \lambda, \quad \lambda, \quad \text { wavelength; }
\end{aligned}
$$$$
n \text {, refractive index of the two media. }
$$

Then we can apply the result in Subsection 2.C. From Eq. (50), we can conclude that the GPSWF for the optical system in Fig. 1 is

$$
\phi_{m}(x) \phi_{n}(y)
$$

where

$$
\begin{aligned}
\phi_{m}(x)= & \left(\begin{array}{l}
k \\
z
\end{array}\right)^{1 / 2} \exp \left\{-j k\left[\frac{1+2 z(n-1) h_{3}}{2 z} x^{2}\right.\right. \\
& \left.\left.+(n-1) h_{1} x\right]\right\} \psi_{m, k B_{1} / z, B_{2}\left(\frac{k}{z} x\right),} \\
\phi_{n}(y)= & \left(\frac{k}{z}\right)^{1 / 2} \exp \left\{-j k\left[\frac{1+2 z(n-1) h_{4}}{2 z} x^{2}\right.\right. \\
& \left.\left.+(n-1) h_{2} x\right]\right\} \psi_{n, k P_{1} / z, P_{2}}\left(\frac{k}{z} y\right),
\end{aligned}
$$

and $\psi_{n, k B_{1} z^{-1}, B_{2}}(x)$ is the original PSWF that satisfies

$$
\begin{aligned}
\int_{-k B_{1} z^{-1}}^{k B_{1} z^{-1}} \frac{\sin \left[B_{2}(\tilde{x}-x)\right]}{\pi(\tilde{x}-x)} \psi_{n, k B_{1} z^{-1}, B_{2}}(x) \mathrm{d} x & \\
& =\lambda_{n} \psi_{n, k B_{1} z^{-1}, B_{2}}(\tilde{x}) .
\end{aligned}
$$

Then we can use the following equation to calculate the energy-preservation ratio:

$$
\begin{aligned}
R= & \frac{\int_{-P_{2}}^{P_{2}} \int_{-B_{2}}^{B_{2}}\left|g_{o}(x, y)\right|^{2} \mathrm{~d} x \mathrm{~d} y}{\int_{-P_{1}}^{P_{1}} \int_{-B_{1}}^{B_{1}}\left|g_{i}(x, y)\right|^{2} \mathrm{~d} x \mathrm{~d} y} \\
= & \frac{\sum_{n=0}^{\infty} \sum_{m=0}^{\infty}\left|\sigma_{m, n}\right|^{2} \lambda_{m}^{2} \lambda_{n}^{2}}{\sum_{n=0}^{\infty} \sum_{m=0}^{\infty}\left|\sigma_{m, n}\right|^{2} \lambda_{m} \lambda_{n}} \\
\sigma_{m, n}= & \lambda_{m}^{-1} \lambda_{n}^{-1} \int_{-P_{1}}^{P_{1}} \int_{-B_{1}}^{B_{1}} g_{i}(x, y) \phi_{m}^{*}(x) \phi_{n}^{*}(y) \mathrm{d} x \mathrm{~d} y .
\end{aligned}
$$

In Fig. 3 we do a numerical simulation to show the energy-preservation conditions for GPSWFs in the optical system as in Fig. 1. The parameters are

$$
\begin{aligned}
& \text { Medium 1. Thickness: } \quad-2.5\left(x^{2}+y^{2}\right)+10^{-2}(x+y) \\
& +8 \times 10^{-3} \\
& \text { extension: } x, y \in[-0.036 \mathrm{~m}, 0.036 \mathrm{~m}] \text {, } \\
& \text { Medium 2. Thickness: }-3.5\left(x^{2}+y^{2}\right)+1.2 \\
& \times 10^{-2}(x+y)+7 \times 10^{-3} \text {; } \\
& \text { extension: } x, y \in[-0.03 \mathrm{~m}, 0.03 \mathrm{~m}] \text {, } \\
& z=1.8 \mathrm{~m}, \lambda=5 \times 10^{-4}, \\
& n=1.5 \text {. }
\end{aligned}
$$

Then the GPSWFs corresponding to the optical system can be obtained from expressions (79) and (80). We can use the process in expressions (12)-(15) to compute $\psi_{m}(x)$

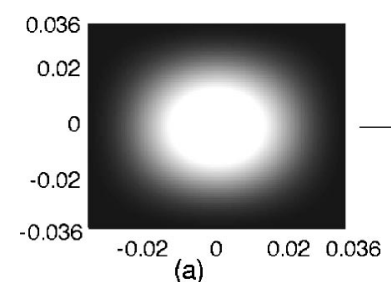

(a)

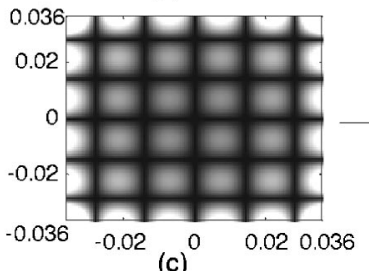

(c)

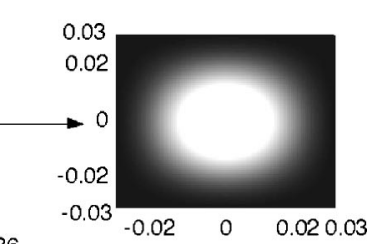

(b)

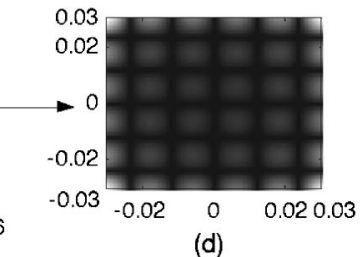

(d)
Fig. 3. Two special orders of the generalized prolate spheroidal functions of the optical system in Fig. 1 . (a) $\phi_{0}(x) \phi_{0}(y)$, (b) output corresponding to $\phi_{0}(x) \phi_{0}(y)$, (c) $\phi_{5}(x) \phi_{5}(y)$, (d) output corresponding to $\phi_{5}(x) \phi_{5}(y)$. 
and $\psi_{n}(x)$ numerically. Then, after multiplication of the quadratic exponential function, the GPSWFs. In Figs. 3(a) and 3(c), we plot two special orders of the GPSWFs, respectively:

$$
\phi_{0}(x) \phi_{0}(y), \quad \phi_{5}(x) \phi_{5}(y),
$$

Then we use the Huygens-Fresnel principle, ${ }^{19}$ which can model an optical system more precisely than the offset fiLCT, to calculate the outputs of the optical system when we use Figs. 3(a) and 3(c) as the inputs. The output results are plotted in Figs. 3(b) and 3(d), respectively. Notice that, except for the differences of intensity and scaling, the intensities of the outputs are almost the same as those of the inputs. It matches property 4 derived in Section 3.

Then we can use Eq. (82) to estimate the powerpreservation ratio. Since

$$
\lambda_{0}=1-5.16 \times 10^{-6}, \quad \lambda_{5}=0.2111,
$$

therefore, from Eq. (82), the power-preservation ratio should be

$$
\text { for } \begin{aligned}
\phi_{0}(x) \phi_{0}(y): \quad R & =\left(1-5.16 \times 10^{-6}\right)^{2} \\
& =1-1.0312 \times 10^{-5}, \\
\text { for } \phi_{5}(x) \phi_{5}(y): \quad R & =0.2111^{2}=0.0446 .
\end{aligned}
$$

We can compare them with the numerical simulation results in Fig. 3. We find that the energy ratio of Fig. 3(b) to Fig. 3(a) is $1-1.0370 \times 10^{-5}$ and the energy ratio of Fig. 3(d) to Fig. 3(c) is 0.0442. Both of them are very close to the estimated power-preservation ratios.

\section{B. Multiple-Stage Case}

In Subsection 4.A we illustrated that we can use GPSWFs to analyze the optical system whose input and output ends are of finite size. However, sometime it may happen that some middle optical components between the input and the output ends are finite sized (usually, except for free spaces, other optical components are of finite size). In this case, it is improper to express the whole system by only one finite transform. We should treat the region between two finite-sized components as a stage, as in Fig. 4, and express each of the stages by the fi-FRFT, the fi-LCT, or the offset fi-LCT. For each stage, we can find the GPSWFs that can be used for analyzing the energypreservation ratio of this stage. Then there is a problem: What is the power eigenfunction of the whole system? We can use the following way to find it.

Assume that there are $Q$ stages and that, for the $q$ th stage, the GPSWFs are $\phi_{q, n}(x)(n=0,1,2, \ldots)$ and the corresponding eigenvalue is $\eta_{q, n}$. If we use $g_{q-1}(x)$ and $g_{q}(x)$ to denote the light distribution at the input and the output of the $q$ th stage and use $\left[T_{q, 1}, T_{q, 2}\right]$ to denote the interval of the $q$ th stage, then $g_{q-1}(x)$ can be expanded as

$$
\begin{aligned}
g_{q-1}(x) & =\sum_{n=0}^{\infty} \sigma_{q, n} \phi_{q, n}(x), \\
\sigma_{q, n} & =\eta_{q, n}^{-1} \int_{T_{q-1,1}}^{T_{q-1,2}} g_{q-1}(x) \phi_{q, n}^{*}(x) \mathrm{d} x .
\end{aligned}
$$

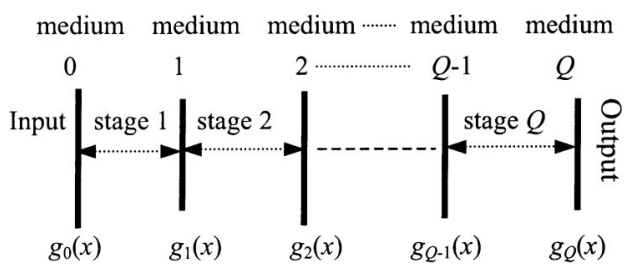

Fig. 4. Finite-sized optical system consisting of multiple stages.

From property 4 in Section 3, we know that $\phi_{q, n}(x)$ is the quasi eigenfunction of the corresponding finite transform. That is, if the $q$ th stage can be represented by the operation $O_{F_{q}}$ (it can be the fi-FRFT, the fi-LCT, or the offset fi-LCT multiplied by some constant phase), then

$$
O_{F_{q}}\left[\phi_{q, n}(x)\right]=\mu_{q, n} \zeta_{q, n}(u) .
$$

For example, from Eq. (73), if the $q$ th stage can be represented by the fi-LCT with parameters $\{a, b, c, d\}$ multiplied by a constant phase $\varphi$ and $x \in\left[T_{1}, T_{2}\right], u$ $\in\left[\Omega_{1}, \Omega_{2}\right]$, then, in Eq. (88),

$$
\begin{aligned}
\phi_{q, n}(x)= & \phi_{n, T_{1}, T_{2}, \Omega_{1}, \Omega_{2}}(x), \\
\zeta_{q, n}(u)= & \mu_{n} k_{1}^{-1 / 2}\left(k_{1}\right)^{-1 / 2} \exp \left(j k_{3} u^{2}\right. \\
& \left.+j k_{4} u\right) \phi_{n, T_{1}, T_{2}, \Omega_{1}, \Omega_{2}}\left[k_{1}^{-1}\left(u-k_{2}\right)\right], \\
\mu_{q, n}= & \exp (j \varphi) \mu_{n},
\end{aligned}
$$

and $k_{1}, k_{2}, k_{3}, k_{4}$, and $\mu_{n}$ are defined in Eqs. (74). Then $g_{q}(u)$, which is the output of the $q$ th stage, can be expressed as

$$
g_{q}(u)=O_{F_{q}}\left[g_{q-1}(x)\right]=\sum_{n=0}^{\infty} \sigma_{q, n} \mu_{q, n} \zeta_{q, n}(u) .
$$

The summation in Eq. (90) is a sum of infinite number of terms. However, owing to the facts that $\left|\mu_{q, n}\right|=\eta_{q, n}^{1 / 2}$ and that $\eta_{q, n}$ is very small when $n$ is large, we can change it into the sums of finite terms. We can choose a threshold $\epsilon$ (near to 0 ) and find $N_{q}$ such that

$$
\eta_{q, n}<\epsilon \quad \text { when } n>N_{q} .
$$

Then Eq. (90) can be approximated by

$$
g_{q}(u) \approx \hat{g}_{q}(u)=\sum_{n=0}^{N_{q}} \sigma_{q, n} \mu_{q, n} \zeta_{q, n}(u) .
$$

Moreover, since the terms $\phi_{q, n}(t)$ have a much smaller effect on $g_{q}(t)$ when $n>N_{q}$, we rewrite Eq. (87) as

$$
\begin{aligned}
\hat{g}_{q-1}(x) & =\sum_{n=0}^{N_{q}} \sigma_{q, n} \phi_{q, n}(x), \\
\sigma_{q, n} & =\eta_{q, n}^{-1} \int_{T_{q-1,1}}^{T_{q-1,2}} g_{q-1}(x) \phi_{q, n}^{*}(x) \mathrm{d} x .
\end{aligned}
$$

Since for each stage the number of effective GPSWFs are finite (i.e., $N_{q}$ 's are finite), we can represent the whole system by matrix operations. First, expressions (92) and (93) can be rewritten as

$$
\hat{g}_{q-1}(x)=\mathbf{p}_{\mathbf{q}} \boldsymbol{\Phi}_{\mathbf{q}}, \quad \hat{g}_{q}(x)=\mathbf{p}_{\mathbf{q}} \boldsymbol{\Lambda}_{\mathbf{q}} \mathbf{Z}_{\mathbf{q}},
$$

where 


$$
\begin{aligned}
& \mathbf{p}_{\mathbf{q}}= {\left[\sigma_{q, 0}, \sigma_{q, 1}, \sigma_{q, 2}, \ldots, \sigma_{q, N_{q}}\right], } \\
& \mathbf{\Phi}_{\mathbf{q}}= {\left[\phi_{q, 0}(x), \phi_{q, 1}(x), \phi_{q, 2}(x), \ldots, \phi_{q, N_{q}}(x)\right]^{T}, } \\
& \mathbf{z}_{\mathbf{q}}= {\left[\zeta_{q, 0}(x), \zeta_{q, 1}(x), \zeta_{q, 2}(x), \ldots, \zeta_{q, N_{q}}(x)\right]^{T}, } \\
& \boldsymbol{\Lambda}_{\mathbf{q}}=\left[\begin{array}{ccccc}
\mu_{q, 0} & 0 & \cdots & 0 & 0 \\
0 & \mu_{q, 1} & \cdots & 0 & 0 \\
\vdots & \vdots & \ddots & \vdots & \vdots \\
0 & 0 & \cdots & \mu_{q, N_{q-1}} & 0 \\
0 & 0 & \cdots & 0 & \mu_{q, N_{q}}
\end{array}\right]
\end{aligned}
$$

$\mu_{q, n}$ is defined in Eq. (88). Moreover, the relation between $\mathbf{Z}_{\mathbf{q}}$ and $\boldsymbol{\Phi}_{\mathbf{q}+\mathbf{1}}$ can be expressed by an $\left(N_{q}+1\right)$ $\times\left(N_{q+1}+1\right)$ matrix $\mathbf{R}_{\mathbf{q}}$ :

$$
\mathbf{Z}_{\mathbf{q}}=\mathbf{R}_{\mathbf{q}} \boldsymbol{\Phi}_{\mathbf{q}+\mathbf{1}},
$$

where

$$
\begin{gathered}
\mathbf{R}_{\mathbf{q}}=\left[\begin{array}{cccc}
r_{q, 0,0} & r_{q, 0,1} & \cdots & r_{q, 0, N_{q+1}} \\
r_{q, 1,0} & r_{q, 1,1} & \cdots & r_{q, 1, N_{q+1}} \\
\vdots & \vdots & \ddots & \vdots \\
r_{q, N_{q}, 0} & r_{q, N_{q}, 1} & \cdots & r_{q, N_{q}, N_{q+1}}
\end{array}\right], \\
r_{q, m, n}=\eta_{q+1, n}^{-1} \int_{T_{q, 1}}^{T_{q, 2}} \zeta_{q, m}(x) \phi_{q+1, n}^{*}(x) \mathrm{d} x .
\end{gathered}
$$

Substituting it into Eqs. (94), we obtain

$$
\hat{g}_{q}(x)=\mathbf{p}_{\mathbf{q}} \boldsymbol{\Lambda}_{\mathbf{q}} \mathbf{Z}_{\mathbf{q}}=\mathbf{p}_{\mathbf{q}} \boldsymbol{\Lambda}_{\mathbf{q}} \mathbf{R}_{\mathbf{q}} \boldsymbol{\Phi}_{\mathbf{q}+\mathbf{1}} .
$$

Then, since $\hat{g}_{q}(x)=\mathbf{p}_{\mathbf{q}+\mathbf{1}} \mathbf{\Phi}_{\mathbf{q}+\mathbf{1}}$,

$$
\mathbf{p}_{\mathbf{q}+\mathbf{1}}=\mathbf{p}_{\mathbf{q}} \Lambda_{\mathbf{q}} \mathbf{R}_{\mathbf{q}}, \quad \mathbf{p}_{\mathbf{Q}}=\mathbf{p}_{1} \Lambda_{1} \mathbf{R}_{1} \Lambda_{2} \mathbf{R}_{2} \cdots \Lambda_{\mathbf{Q}-1} \mathbf{R}_{\mathbf{Q}-\mathbf{1}} .
$$

Thus, for the multiple-stage optical system in Fig. 4, the relation between the input $g_{0}(x)$ and the output $g_{Q}(x)$ can be expressed as

$$
\begin{aligned}
\text { input: } g_{0}(x) & =\sum_{n=0}^{\infty} \sigma_{1, n} \phi_{1, n}(x), \\
\text { output: } g_{Q}(x) & \approx \sum_{n=0}^{N_{Q}} \beta_{n} \zeta_{Q, n}(x), \\
\mathbf{p}_{\mathbf{1}} & =\left[\sigma_{q, 0}, \sigma_{q, 1}, \sigma_{q, 2}, \ldots, \sigma_{q, N_{1}}\right], \\
\mathbf{p}_{\mathbf{o}} & =\left[\beta_{0}, \beta_{1}, \beta_{2}, \ldots, \beta_{N_{Q}}\right], \\
\mathbf{p}_{\mathbf{o}} & =\mathbf{p}_{\mathbf{1}} \mathbf{A}, \\
\mathbf{A} & =\boldsymbol{\Lambda}_{\mathbf{1}} \mathbf{R}_{\mathbf{1}} \mathbf{\Lambda}_{\mathbf{2}} \mathbf{R}_{\mathbf{2}} \cdots \boldsymbol{\Lambda}_{\mathbf{Q}-\mathbf{1}} \mathbf{R}_{\mathbf{Q}-\mathbf{1}} \boldsymbol{\Lambda}_{\mathbf{Q}} .
\end{aligned}
$$

$$
\begin{aligned}
R & =\frac{\int_{T_{Q, 1}}^{T_{Q, 2}}\left|g_{Q}(u)\right|^{2} \mathrm{~d} u}{\int_{T_{0,1}}^{T_{0,2}}\left|g_{0}(x)\right|^{2} \mathrm{~d} x} \approx \frac{\sum_{n=0}^{T_{Q}}\left|\mu_{n}\right|^{2} \eta_{Q, n}}{\sum_{n=0}^{\infty}\left|\sigma_{1, n}\right|^{2} \eta_{1, n}} \\
= & C \frac{\sum_{n=0}^{T_{Q}}\left|\mu_{n}\right|^{2} \eta_{Q, n}}{\sum_{n=0}^{T_{1}}\left|\sigma_{1, n}\right|^{2} \eta_{1, n}}=C \frac{\mathbf{p}_{\mathbf{o}}\left|\boldsymbol{\Lambda}_{\mathbf{Q}}\right|^{2} \mathbf{p}_{\mathbf{o}}^{\mathbf{H}}}{\mathbf{p}_{\mathbf{1}}\left|\mathbf{\Lambda}_{\mathbf{1}}\right|^{2} \mathbf{p}_{\mathbf{1}}^{\mathbf{H}}} \\
= & C \frac{\mathbf{p}_{\mathbf{1}} \mathbf{A}\left|\boldsymbol{\Lambda}_{\mathbf{Q}}\right|^{2} \mathbf{A}^{\mathbf{H}} \mathbf{p}_{\mathbf{1}}^{\mathbf{H}}}{\mathbf{p}_{\mathbf{1}}\left|\mathbf{\Lambda}_{\mathbf{1}}\right|^{2} \mathbf{p}_{\mathbf{1}}^{\mathbf{H}}}=C \frac{\mathbf{p}_{\Delta}\left|\mathbf{\Lambda}_{\mathbf{1}}\right|^{-1} \mathbf{A}\left|\mathbf{\Lambda}_{\mathbf{Q}}\right|^{2} \mathbf{A}^{\mathbf{H}}\left|\mathbf{\Lambda}_{\mathbf{1}}\right|^{-1} \mathbf{p}_{\Delta}^{\mathbf{H}}}{\mathbf{p}_{\Delta} \mathbf{p}_{\Delta}^{\mathbf{H}}},
\end{aligned}
$$

where

$$
\begin{aligned}
C & =\sum_{n=0}^{T_{1}}\left|\sigma_{1, n}\right|^{2} \eta_{1, n}\left(\sum_{n=0}^{\infty}\left|\sigma_{1, n}\right|^{2} \eta_{1, n}\right)^{-1}, \\
\sigma_{1, n} & =\eta_{1, n}^{-1} \int_{T_{0,1}}^{T_{0,2}} g_{0}(x) \phi_{1, n}^{*}(x) \mathrm{d} x, \\
\mathbf{p}_{\Delta} & =\mathbf{p}_{\mathbf{1}}\left|\boldsymbol{\Lambda}_{\mathbf{1}}\right| .
\end{aligned}
$$

We can use the method of eigenvector decomposition to simplify expression (101). Assume that

$$
\mathbf{B}=\left|\boldsymbol{\Lambda}_{\mathbf{1}}\right|^{-1} \mathbf{A}\left|\boldsymbol{\Lambda}_{\mathbf{Q}}\right|^{2} \mathbf{A}^{\mathbf{H}}\left|\boldsymbol{\Lambda}_{\mathbf{1}}\right|^{-1}, \quad \mathbf{f}_{\mathbf{n}} \mathbf{B}=\kappa_{n} \mathbf{f}_{\mathbf{n}} .
$$

Notice that $\mathbf{B}$ is an $\left(N_{1}+1\right) \times\left(N_{1}+1\right)$ matrix and $\mathbf{f}_{\mathbf{n}}\left(n=0 \sim N_{1}\right)$ is an eigenvector of $\mathbf{B}$. The size of $\mathbf{f}_{\mathbf{n}}$ is $1 \times\left(N_{1}+1\right)$. Owing to the fact that $\mathbf{B}$ is the product of a matrix and its Hermitian form (i.e., $B=\tilde{\mathbf{A}} \tilde{\mathbf{A}}^{\mathbf{H}}$, where $\widetilde{\mathbf{A}}=\left|\boldsymbol{\Lambda}_{\mathbf{1}}\right|^{-1} \mathbf{A}\left|\boldsymbol{\Lambda}_{\mathbf{Q}}\right|$ ), the eigenvalues $\kappa_{n}$ 's are real and positive. Moreover, since $\mathbf{B}^{\mathbf{H}}=\mathbf{B}$, the eigenvectors $\mathbf{f}_{\mathbf{n}}$ 's are orthogonal (i.e., $\mathbf{f}_{\mathbf{m}} \mathbf{f}_{\mathbf{n}}^{\mathbf{H}}=0$ if $m \neq n$ ). We can sort $\mathbf{f}_{\mathbf{n}}$ according to $\kappa_{n}$,

$$
1>\kappa_{0}>\kappa_{1}>\kappa_{2}>\cdots>\kappa_{N_{1}}>0,
$$

and normalize $\mathbf{f}_{\mathbf{n}}$ such that $\mathbf{f}_{\mathbf{n}} \mathbf{f}_{\mathbf{n}}^{\mathbf{H}}=1$. Then $\mathbf{p}_{\Delta}$ can be expanded as

$$
\mathbf{p}_{\Delta}=\sum_{n=0}^{N_{1}} \alpha_{n} \mathbf{f}_{\mathbf{n}}, \quad \alpha_{n}=\mathbf{p}_{\Delta} \mathbf{f}_{\mathbf{n}}^{\mathbf{H}}=\mathbf{p}_{\mathbf{1}}\left|\boldsymbol{\Lambda}_{\mathbf{1}}\right| \mathbf{f}_{\mathbf{n}}^{\mathbf{H}},
$$

and expression (101) can be rewritten as

$$
\begin{aligned}
& R=C \frac{\mathbf{p}_{\Delta} \mathbf{B} \mathbf{p}_{\Delta}^{\mathbf{H}}}{\mathbf{p}_{\Delta} \mathbf{p}_{\Delta}^{\mathbf{H}}}=C \frac{\sum_{n=0}^{N_{1}} \sum_{m=0}^{N_{1}} \alpha_{n} \alpha_{m}^{*} \mathbf{f}_{\mathbf{n}} \mathbf{B} \mathbf{f}_{\mathbf{m}}^{\mathbf{H}}}{\sum_{n=0}^{N_{1}} \sum_{m=0}^{N_{1}} \alpha_{n} \alpha_{m}^{*} \mathbf{f}_{\mathbf{n}} \mathbf{f}_{\mathbf{m}}^{\mathbf{H}}} \\
& =C \frac{\sum_{n=0}^{N_{1}} \sum_{m=0}^{N_{1}} \alpha_{n} \alpha_{m}^{*} \kappa_{n} \mathbf{f}_{\mathbf{n}} \mathbf{f}_{\mathbf{m}}^{\mathbf{H}}}{\sum_{n=0}^{N_{1}} \sum_{m=0}^{N_{1}} \alpha_{n} \alpha_{m}^{*} \mathbf{f}_{\mathbf{n}} \mathbf{f}_{\mathbf{m}}^{\mathbf{H}}}=C \frac{\sum_{n=0}^{N_{1}}\left|\alpha_{n}\right|^{2} \kappa_{n}}{\sum_{n=0}^{N_{1}}\left|\alpha_{n}\right|^{2}},
\end{aligned}
$$

So the energy-preservation ratio can be calculated from 
where C is defined in Eqs. (102). We can use Eq. (106) to calculate the power-preservation ratio of the multiplestage optical system in Fig. 4. Specially, when

$$
\mathbf{p}_{\Delta}=\alpha_{0} \mathbf{f}_{\mathbf{0}}, \quad \text { i.e., } \mathbf{p}_{\mathbf{1}}=\alpha_{0} \mathbf{f}_{\mathbf{0}}\left|\boldsymbol{\Lambda}_{\mathbf{1}}\right|^{-1},
$$

the power-preservation ratio $R$ is maximal. In this case,

$$
R=C \kappa_{0} .
$$

\section{Analyzing Self-Imaging Phenomena}

Not only can we use GPSWFs to analyze the powerpreservation ratio of the finite-sized optical system, but we can also use it to analyze the self-imaging phenomenon. If the effect of the finite size of optical components is ignored, we can use the eigenfunctions of the FT, the FRFT, the LCT, and the offset LCT to analyze the selfimaging phenomenon of the optical system. ${ }^{17}$ However, to analyze the self-imaging phenomenon of the finitesized optical component more precisely, it is proper to use GPSWFs instead of the eigenfunctions of the original nonfinite transforms.

To show how GPSWFs cause self-imaging phenomena for finite-sized optical systems, we can apply property 4 in Section 3. From Eq. (73), we know that, although GPSWFs may not be the eigenfunction of the offset fiLCT, it can be viewed as the quasi eigenfunction of the offset fi-LCT. In fact, Eq. (73) can be rewritten as

$$
\begin{aligned}
O_{\widetilde{F}}^{(a, b, c, d, \tau, \rho)}\left[\phi_{n}(x)\right]= & \mu_{n} \zeta_{n}(u), \\
\zeta_{n}(u)= & \left(k_{1}\right)^{-1 / 2} \exp \left(j k_{3} u^{2}\right. \\
& \left.+j k_{4} u\right) \phi_{n}\left(\frac{u-k_{2}}{k_{1}}\right) .
\end{aligned}
$$

Notice that $\zeta_{n}(x)$ and $\phi_{n}(x)$ differ only in phase, scaling, and shifting. Assume that an optical system can be represented by the offset fi-LCT with parameters $\{a, b, c, d, \tau, \rho\}$ :

$$
\text { Optical system: } f_{o}(x)=\exp (j \varphi) O_{\widetilde{F}}^{(a, b, c, d, \tau, \rho)}\left[f_{i}(x)\right],
$$

$$
f_{i}(x) \text { : input, } f_{o}(x) \text { : output. }
$$

If the distribution of the input light is the GPSWF multiplied by some constant, i.e., $f_{i}(x)=C \phi_{n}(x)$, then

$$
f_{o}(x)=\exp (j \varphi) C \mu_{n} \zeta_{n}(x) .
$$

In optics, we only observe the intensity of light. The intensity of $f_{o}(x)$ is

$$
\begin{aligned}
\left|f_{o}(x)\right| & =\left|C \mu_{n} \zeta_{n}(x)\right|=\left(k_{1}^{-1} \eta_{n}\right)^{1 / 2}\left|C \phi_{n}\left(\frac{x-k_{2}}{k_{1}}\right)\right| \\
& =\left(k_{1}^{-1} \eta_{n}\right)^{1 / 2}\left|f_{i}\left(\frac{x-k_{2}}{k_{1}}\right)\right| .
\end{aligned}
$$

Thus the output intensity is the scaling and space shifting of that of $f_{i}(x)$ multiplied by some constant. The input light distribution $\left|f_{i}(x)\right|$ appears again at the output, and the self-imaging phenomenon was formed.

Therefore, although the GPSWF $\phi_{n}(x)$ is not the eigenfunction of the offset fi-LCT, it can cause the self-imaging phenomenon for the optical system that can be modeled by the offset fi-LCT.
In addition to GPSWFs, the linear combination of GPSWFs with almost the same values of $\mu_{n}$ can also cause the self-imaging phenomenon. Strictly speaking, the eigenvalues of the GPSWFs are different from one another (i.e., $\mu_{n} \neq \mu_{m}$ if $n \neq m$ ). However, if the product of $T$ and $\Omega$ is not too small, there are several GPSWFs whose values of $\left|\mu_{n}\right|=\eta_{n}^{1 / 2}$ are close to 1 :

$$
\begin{aligned}
& 1>\left|\mu_{n}\right|>1-\Delta ; \\
& 0 \leqslant n \leqslant \tilde{N}, \quad \Delta \text { very small. }
\end{aligned}
$$

In other words, in Eqs. (74), $\left|\mu_{n}\right| \approx 1$ when $n \leqslant \widetilde{N}$ and

$$
\begin{gathered}
\mu_{n} \approx \mu_{m} \approx j^{-(n+1 / 2) \operatorname{sgn}(b)} \exp (j \theta), \quad m, n \leqslant \tilde{N}, \\
m-n=4 M, \quad \text { where } M \text { is an integer, } \\
\theta=b^{-1}\left[2^{-1} d \tau^{2}+\Omega_{0} T_{0}+2^{-1} a k_{1}^{-2} k_{2}^{2}\right. \\
\left.\quad+k_{1}^{-1} k_{2}\left(\Omega_{0}-\tau\right)\right] .
\end{gathered}
$$

Therefore, for the finite-sized optical system in Eq. (110), if the input light distribution is the linear combination of the GPSWFs as follows,

$$
\begin{aligned}
f_{i}(x) & =\sum_{s=0}^{N_{s}} \alpha_{s} \phi_{r+4 s}(x), \quad r=0,1,2, \text { or } 3, \\
r+4 N_{s} & \leqslant \tilde{N}<r+4\left(N_{s}+1\right),
\end{aligned}
$$

then

$$
\begin{aligned}
f_{o}(u) & =\exp (j \varphi) O_{\widetilde{F}}^{(a, b, c, d, \tau, \rho)}\left[\sum_{s=0}^{N_{s}} \alpha_{s} \phi_{r+4 s}(x)\right] \\
& =\exp (j \varphi) \sum_{s=0}^{N_{s}} \alpha_{s} \mu_{r+4 s} \zeta_{r+4 s}(x) \\
& \approx \exp [j(\varphi+\theta)] j^{-(n+1 / 2) \operatorname{sgn}(b)} \sum_{s=0}^{N_{s}} \alpha_{s} \zeta_{r+4 s}(u) \\
& =k_{1}^{-1 / 2}\left|\sum_{s=0}^{N_{s}} \alpha_{s} \phi_{r+4 s}\left[k_{1}^{-1}\left(u-k_{2}\right)\right]\right| \\
\left|f_{o}(u)\right| & \approx k_{1}^{-1 / 2}\left|f_{i}\left(\frac{u-k_{2}}{k_{1}}\right)\right|
\end{aligned}
$$

where $k_{1}$ and $k_{2}$ are defined in Eqs. (74).

Thus the intensity of the output is close to the scaling and space shifting of the intensity of the input light, and the linear combination of the GPSWFs as in Eq. (115) is also able to cause the self-imaging phenomenon with a little distortion.

\section{Resonance Phenomenon}

It has been known that we can use the eigenfunctions of the LCT and the offset LCT to analyze the resonance phenomenon for the spherical-mirror-pair system in Fig. $5 .{ }^{17}$ However, in practice, the size of the mirror-pair system is finite. To obtain more precise results, it is proper to use GPSWFs instead of the eigenfunctions of the LCT and the offset LCT to analyze resonance phenomena. 


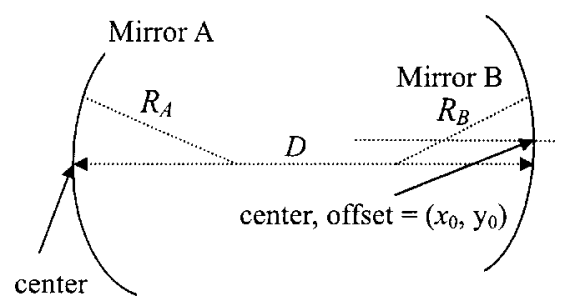

Fig. 5. Spherical-mirror-pair system.

Assume that the extensions of the two spherical mirrors in Fig. 5 are

$$
\begin{aligned}
& \text { mirror A: } \quad x, y \in\left[-B_{1}, B_{1}\right] \\
& \text { mirror B: } x, y \in\left[-B_{2}, B_{2}\right]
\end{aligned}
$$

If the light distribution emitted from mirror $\mathrm{A}$ is $f_{i, 0}(x, y)$ and the light accepted by mirror $\mathrm{B}$ is $f_{o, 0}(x, y)$, then the relation between $f_{i, 0}(x, y)$ and $f_{o, 0}(x, y)$ can be expressed by the offset fi-LCT:

$$
\begin{aligned}
f_{o, 0}(x, y)= & \exp \left(j \varphi_{1}\right) O_{\tilde{F}_{y}}^{\left(a_{1}, b_{1}, c_{1}, d_{1}, \tilde{\tau}_{1}, \tilde{\rho}_{1}\right)}\left\{O_{\tilde{F}_{x}}^{\left(a_{1}, b_{1}, c_{1}, d_{1}, \tau_{1}, \rho_{1}\right)}\right. \\
& \left.\times\left[f_{i, 0}(x, y)\right]\right\},
\end{aligned}
$$

where $O_{\tilde{F}_{x}}$ and $O_{\tilde{F}_{y}}$ means the one-dimensional offset fiLCTs along the $x$ axis and $y$ axis, respectively, $\varphi_{1}$ is some constant phase, and

$$
\begin{aligned}
{\left[\begin{array}{ll}
a_{1} & b_{1} \\
c_{1} & d_{1}
\end{array}\right] } & =\left[\begin{array}{cc}
1-R_{1}^{-1} D & -D / k \\
k\left(R_{1}^{-1}+R_{2}^{-1}-R_{1}^{-1} R_{2}^{-1} D\right) & 1-R_{2}^{-1} D
\end{array}\right], \\
k & =2 \pi / \lambda, \quad \tau_{1}=\tilde{\tau}_{1}=0 \\
\rho_{1} & =-k x_{0} / R_{2}, \quad \tilde{\rho}_{1}=-k y_{0} / R_{2}
\end{aligned}
$$

$D$ is the distance between the two mirrors, and $R_{1}$ and $R_{2}$ are the radii of mirrors $A$ and $B$. After the light reaches mirror $\mathrm{B}$, it is reflected and propagates back to mirror $\mathrm{A}$. Assume that the reflective light accepted by mirror $\mathrm{A}$ is $f_{i, 1}(x, y)$. The relation between $f_{i, 1}(x, y)$ and $f_{o, 0}(x, y)$ is

$$
\begin{aligned}
f_{i, 1}(x, y)= & \exp \left(j \varphi_{2}\right) O_{\widetilde{F}_{y}}^{\left(a_{2}, b_{2}, c_{2}, d_{2}, \tilde{\tau}_{2}, \tilde{\rho}_{2}\right)}\left\{O_{\widetilde{F}_{x}}^{\left(a_{2}, b_{2}, c_{2}, d_{2}, \tau_{2}, \rho_{2}\right)}\right. \\
& \left.\times\left[f_{o, 0}(x, y)\right]\right\}
\end{aligned}
$$

where $\varphi_{2}$ is some constant phase and

$$
\begin{aligned}
{\left[\begin{array}{ll}
a_{2} & b_{2} \\
c_{2} & d_{2}
\end{array}\right] } & =\left[\begin{array}{cc}
1-R_{2}^{-1} D & -D / k \\
k\left(R_{1}^{-1}+R_{2}^{-1}-R_{1}^{-1} R_{2}^{-1} D\right) & 1-R_{1}^{-1} D
\end{array}\right], \\
\left(\begin{array}{c}
\tau_{2} \\
\rho_{2}
\end{array}\right) & =\left[\begin{array}{c}
x_{0} D R_{2}^{-1} \\
k x_{0} R_{2}^{-1}\left(R_{1}^{-1} D-1\right)
\end{array}\right], \\
\left(\begin{array}{c}
\tilde{\tau}_{2} \\
\tilde{\rho}_{2}
\end{array}\right) & =\left[\begin{array}{c}
y_{0} D R_{2}^{-1} \\
k y_{0} R_{2}^{-1}\left(R_{1}^{-1} D-1\right)
\end{array}\right] .
\end{aligned}
$$

In the case where

$$
f_{i, 1}(x, y)=\rho f_{i, 0}(x, y), \quad \rho \text { is some constant, }
$$

the resonance phenomenon forms. Then we discuss under what condition Eq. (122) is satisfied. In fact, this problem is similar to the multiple-stage problem dis- cussed in Subsection 4.B. The propagation from mirror A to mirror $\mathrm{B}$ can be treated as stage 1 , and the propagation from mirror B back to mirror A can be treated as stage 2 . If the input is $f_{i, 0}(x, y)$, we can use the method as in expressions (100) to determine the output $f_{i, 1}(x, y)$. Notice that in this case $Q=2$. It can be shown that Eq. (122) is satisfied if

$$
f_{i, 0}(x, y)=\left[\sum_{n=0}^{N_{1}} \sigma_{1, n} \phi_{1, n}(x)\right]\left[\sum_{n=0}^{N_{1}} \sigma_{1, n}^{\prime} \phi_{1, n}(y)\right],
$$

where $\quad \mathbf{e}_{\mathbf{m}}=\left[\sigma_{m, 0}, \sigma_{m, 1}, \sigma_{m, 2}, \ldots, \sigma_{m, N_{1}}\right]^{\mathrm{T}}$ and $\mathbf{e}_{\mathbf{m}}^{\prime}$ $=\left[\sigma_{m, 0}^{\prime}, \sigma_{m, 1}^{\prime}, \sigma_{m, 2}^{\prime}, \ldots, \sigma_{m, N_{1}}^{\prime}\right]^{\mathrm{T}}$ are the eigenvectors of $\mathbf{D}=\Lambda_{1} \mathbf{R}_{1} \Lambda_{2} \mathbf{R}_{2} \mathbf{H}$,

$$
\begin{aligned}
\mathbf{H} & =\left[\begin{array}{cccc}
h_{0,0} & h_{0,1} & \cdots & h_{0, N_{2+1}} \\
h_{1,0} & h_{1,1} & \cdots & h_{1, N_{2+1}} \\
\vdots & \vdots & \ddots & \vdots \\
h_{N_{2}, 0} & h_{N_{2}, 1} & \cdots & h_{N_{2}, N_{2+1}}
\end{array}\right], \\
h_{m, n} & =\eta_{1, n}^{-1} \int_{-B_{1}}^{B_{1}} \zeta_{2, m}(x) \phi_{1, n}^{*}(x) \mathrm{d} x,
\end{aligned}
$$

and $\Lambda_{\mathbf{q}}, \mathbf{R}_{\mathbf{q}}, \phi_{q, n}(x)$, and $\zeta_{q, n}(x)$ were defined in Subsection 4.B. In this case, the resonance phenomenon occurs.

\section{CONCLUSIONS}

In the literature the PSWFs for the fi-FT were derived. In this paper we extend the previous studies and derived the GPSWFs for the fi-FRFT (a generalization of the fiFT), the fi-LCT (a further generalization of the fi-LCT), and the offset fi-LCT (the fi-LCT with two extra offset parameters). In addition, in the literature, PSWFs were often used for analyzing the case where the extension is centered at zero. In this paper, with the results in Subsection 2.D, we can analyze the case where the interval is not symmetric about zero.

Since the GPSWFs corresponding to the fi-FRFT, the fiLCT, and the offset fi-LCT are derived, and many optical systems can be represented by these three finite operations, some theory about finite-sized optical system analysis can be developed. We can use the GPSWFs to analyze the energy-preservation ratio, the self-imaging phenomenon, and the resonance phenomenon of the finite-sized optical system.

\section{ACKNOWLEDGMENT}

The research was supported in part by the National Science Council of Taiwan, under contracts NSC 93-2811-E002-046 and NSC93-2752-E-002-006-PAE.

S.-C. Pei, the corresponding author, can be reached by fax, 886-2-23671909, or by e-mail, pei@cc.ee.ntu.edu.tw. 


\section{REFERENCES}

1. D. Slepian and H. O. Pollak, "Prolate spheroidal wave functions, Fourier analysis and uncertainty-I," Bell Syst. Tech. J. 40, 43-63 (1961).

2. H. J. Landau and H. O. Pollak, "Prolate spheroidal wave functions, Fourier analysis and uncertainty-II," Bell Syst. Tech. J. 40, 65-84 (1961).

3. H. J. Landau and H. O. Pollak, "Prolate spheroidal wave functions, Fourier analysis and uncertainty-III," Bell Syst. Tech. J. 41, 1295-1336 (1962).

4. D. Slepian, "Some asymptotic expansions for prolate spheroidal wave functions," J. Math. Phys. 44, 99-140 (1965).

5. D. Slepian, "Prolate spheroidal wave functions, Fourier analysis, and uncertainty-V: the discrete case," Bell Syst. Tech. J. 57, 1371-1430 (1977).

6. J. H. Wilkinson, The Algebraic Eigenvalue Problem (Oxford U. Press, London, 1988).

7. B. R. Frieden, "Evaluation, design and extrapolation methods for optical signals, based on use of the prolate functions," in Progress in Optics, Vol. IX, E. Wolf, ed. (NorthHolland, Amsterdam, 1971), pp. 311-407.

8. A. Papoulis and M. S. Bertran, "Digital filtering and prolate functions," IEEE Trans. Circuit Theory 19, 674-681 (1972).

9. H. M. Ozaktas, M. A. Kutay, and Z. Zalevsky, The Fractional Fourier Transform with Applications in Optics and Signal Processing (Wiley, New York, 2000).
10. V. Namias, "The fractional order Fourier transform and its application to quantum mechanics," J. Inst. Math. Appl. 25, 241-265 (1980).

11. K. B. Wolf, Integral Transforms in Science and Engineering (Plenum, New York, 1979), Chap. 9, pp. 381-416.

12. L. M. Bernardo, " $A B C D$ matrix formalism of fractional Fourier optics," Opt. Eng. (Bellingham) 35, 732-740 (1996).

13. M. J. Bastiaans, "Propagation laws for the secondorder moments of the Wigner distribution function in firstorder optical systems," Optik (Stuttgart) 82, 173-181 (1989).

14. H. M. Ozaktas and D. Mendlovic, "Fractional Fourier optics," J. Opt. Soc. Am. A 12, 743-751 (1995).

15. P. Pellat-Finet and G. Bonnet, "Fractional order Fourier transform and Fourier optics," Opt. Commun. 111, 141-154 (1994).

16. S. Abe and J. T. Sheridan, "Optical operations on wave functions as the Abelian subgroups of the special affine Fourier transformation," Opt. Lett. 19, 1801-1803 (1994).

17. S. C. Pei and J. J. Ding, "Eigenfunctions of the offset Fourier, fractional Fourier, and linear canonical transforms," J. Opt. Soc. Am. A 20, 522-532 (2003).

18. K. Khare and N. George, "Fractional finite Fourier transform," J. Opt. Soc. Am. A 21, 1179-1185 (2004).

19. J. W. Goodman, Introduction to Fourier Optics (McGrawHill, New York, 1996). 